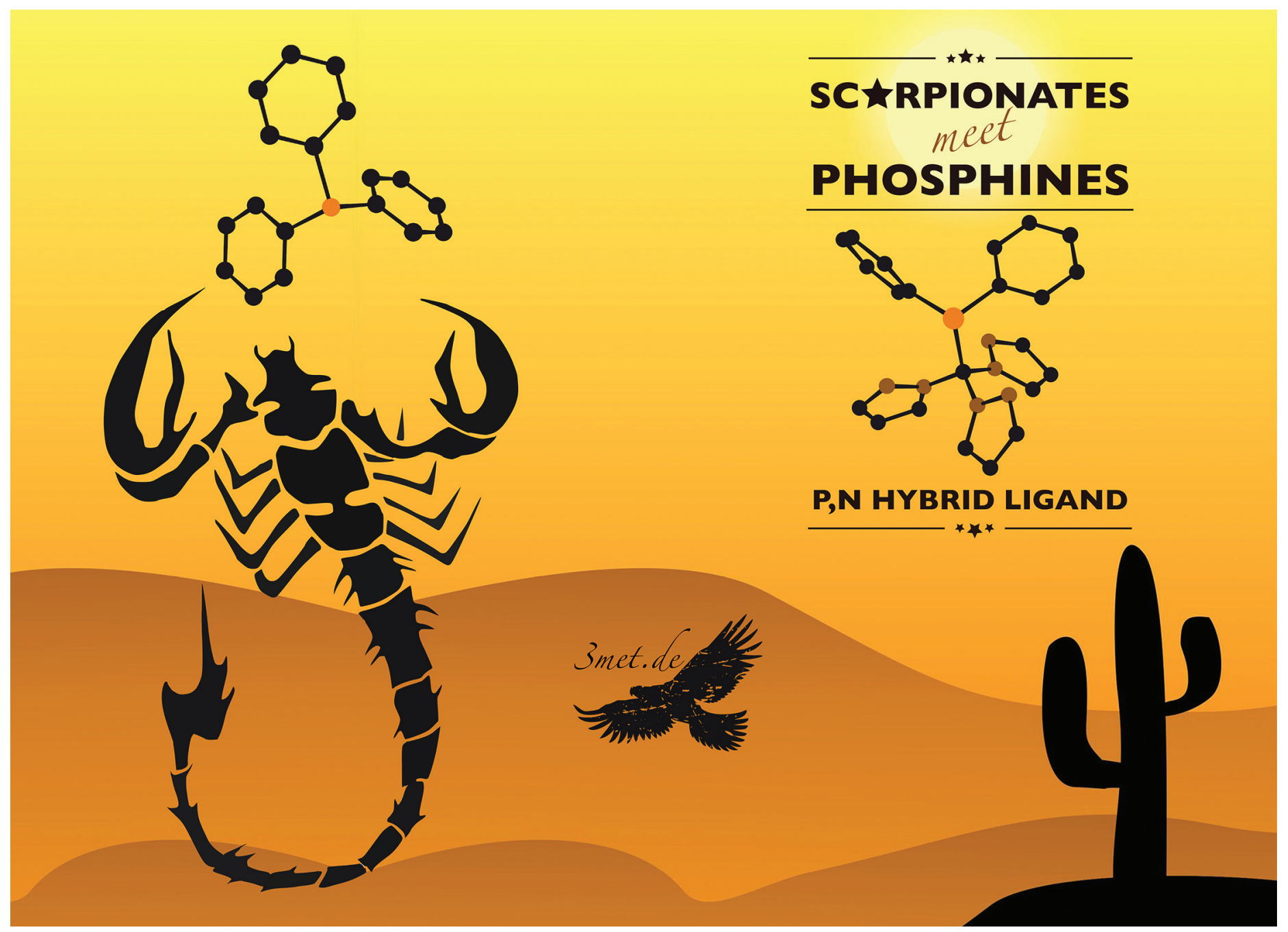

Showcasing research from the group of Frank Breher from the Karlsruhe Institute of Technology (KIT).

Phosphine-functionalised tris(pyrazolyl)methane ligands and their mono- and heterobimetallic complexes

The synthesis, characterisation and reactivity of phosphinefunctionalised tris(pyrazolyl)methane ligands are presented. The new P,N hybrid ligands feature characteristics of both "classical" scorpionates and phosphine ligands. Their coordination flexibility and their ability to generate heterobimetallic complexes has been demonstrated.

\section{As featured in:}

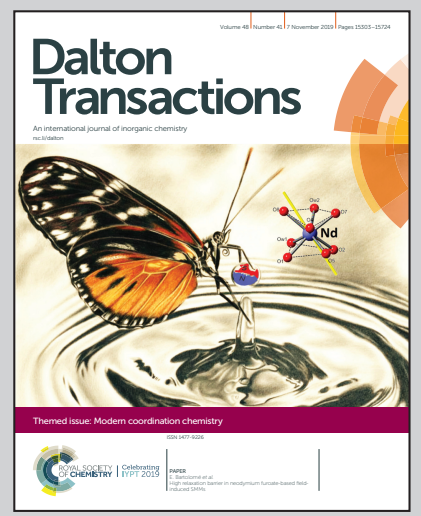

See Frank Breher et al., Dalton Trans., 2019, 48, 15397. 


\section{D) Check for updates}

Cite this: Dalton Trans., 2019, 48, 15397

Received 16th May 2019,

Accepted 5th June 2019

DOI: 10.1039/c9dt02057h

rsc.li/dalton

\title{
Phosphine-functionalised tris(pyrazolyl)methane ligands and their mono- and heterobimetallic complexes $\uparrow+$
}

\author{
Hanna E. Wagner, Silvia Hohnstein, Max G. Schußmann, Lukas A. Steppe and \\ Frank Breher (DD *
}

\begin{abstract}
The synthesis, characterisation and reactivity of new phosphine-functionalised tris(pyrazolyl)methane ligands $\left(\mathrm{TpmPR}_{2}, \mathbf{2} \mathbf{a}-\mathbf{c}\right.$, with $\mathrm{R}=\mathrm{Ph}, n \mathrm{Bu}$, iPr) are presented. The reaction of $\mathbf{2 a}-\mathbf{c}$ with $\left[\mathrm{Rh}(\mathrm{CO})_{2} \mathrm{Cl}_{2}\right.$ furnished $N, P$-heterochelate carbonyl complexes $(3 a-c)$, which were used to quantify the donor abilities of the ligands via IR spectroscopy. The coordination flexibility was demonstrated by treating representative members of this new ligand class with $\left[\mathrm{CpRu}(\mathrm{acn})_{3}\right]\left[\mathrm{PF}_{6}\right]$ (acn = acetonitrile), [(tht)AuCl] (tht = tetrahydrothiophene) and $\left[\mathrm{Pd}(\mathrm{allyl}) \mathrm{Cl}_{2}\right.$ providing either a $\mathrm{N}, \mathrm{N}, \mathrm{P}$-heteroscorpionate complex (4) or the $P$-coordinated complexes $(\mathbf{5 , 6})$ without any involvement of the pyrazolyl entities. With $\mathbf{2 a}$ and $\left[\mathrm{Pd}(\mathrm{cod}) \mathrm{Cl}_{2}\right]$, another $\mathrm{P}, \mathrm{N}$-heterochelate complex (7) was obtained, which served as a precursor for a heterobimetallic complex containing palladium and copper (8). Detailed NMR spectroscopic and X-ray crystallographic investigations have been performed on all new complexes.
\end{abstract}

\section{Introduction}

Multidentate ligand systems have been of interest for several decades for the generation of transition metal complexes with specific coordination numbers, nuclearities, or structures. In particular, tripodal fac-coordinating N-donor ligands such as tris(pyrazolyl)hydroborates $\left(\mathrm{Tp}^{\mathrm{R}}\right)^{1}$ or tris(pyrazolyl)methanes $\left({ }^{\mathrm{R}} \mathrm{Tpm}\right)^{2}$ provide intrinsically well-defined coordination geometries. Scorpionate ligands of this type have long been of interest in the area of bioinorganic, organometallic or coordination chemistry. ${ }^{1-3}$ This ligand family has been extended some years ago to the anionic tris(pyrazolyl)methanide ${ }^{4}$ and -silanide ${ }^{4 i, 5}$ systems, ${ }^{6}$ which are isoelectronic with the hydroborate ligands, but feature ambidentate characteristics and two possible binding sites, i.e. the anionic carbon and silicon or the pyrazolyl ring nitrogen donors. Another possibility for increasing the number of donor atoms in scorpionate-type ligand scaffolds is to attach further donor-containing entities either in the 3-position of the pyrazolyl entities ${ }^{7}$ or at the apical boron $\left(\mathrm{Tp}^{\mathrm{R}}\right)$ or carbon $\left({ }^{\mathrm{R}} \mathrm{Tpm}\right)$ unit. Ligand systems that are functionalised at the non-coordinating back position of the scorpionate are

Institute of Inorganic Chemistry, Karlsruhe Institute of Technology (KIT), Engesserstr. 15, 76131 Karlsruhe, Germany. E-mail: breher@kit.edu

$\dagger$ Dedicated to Prof. Annie Powell on the occasion of her 60th birthday.

\$Electronic supplementary information (ESI) available: NMR data, X-ray crystal structure data. CCDC 1916045-1916055. For ESI and crystallographic data in CIF or other electronic format see DOI: 10.1039/c9dt02057h commonly referred to as "third generation scorpionates". As recently stated by Reger et al. in a comprehensive review article $^{8}$ the tris(pyrazolyl)methane ligands are particularly well suited for these studies as the chemistry is based on reaction chemistry at a $\mathrm{C}-\mathrm{H}$ bond. Functional groups attached to this apical position can either affect the properties of the ligand, like its solubility, or increase the number of possible coordination modes. ${ }^{9}$ Selected examples of backbone-functionalised Tpm hybrid ligands are compiled in Chart 1.

As part of our investigations on ambidentate ligands, ${ }^{10}$ we pursued the introduction of additional donor functions as additional binding sites in a Tpm-based ligand framework. We

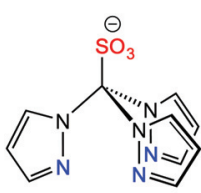

Kläui (2000)<smiles>OCC(n1cccn1)n1cccn1</smiles>

Reger (2000)<smiles></smiles>

Mews (2001)

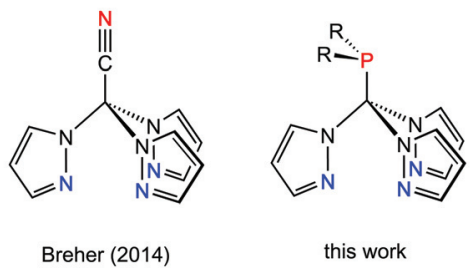

Chart 1 Backbone-functionalised Tpm ligands from the literature. 
report here on neutral, backbone-functionalised Tpm hybrid ligands, ${ }^{11}$ namely tris( pyrazolyl)methyl phosphines. The latter were found to be versatile hybrid ligands for mono- and heterobimetallic transition metal complexes.

\section{Results and discussion}

Synthesis and characterisation of $\operatorname{TpmPR}_{2}(\mathrm{R}=\mathrm{Ph}, n \mathrm{Bu}, \mathrm{iPr})$

Inspired by the pioneering work of Otero ${ }^{12}$ and Burzlaff ${ }^{13}$ on heteroscorpioante ligands based on bis(pyrazolyl)methanes ${ }^{2 b}$ or Reger ${ }^{9 d}$ and Kläui ${ }^{9 a}$ on tris(pyrazolyl)methanes, freshly prepared lithium tris(pyrazolyl)methanide (1) was reacted with $\mathrm{R}_{2} \mathrm{PCl}$. Moisture and air sensitive colourless crystalline pro-

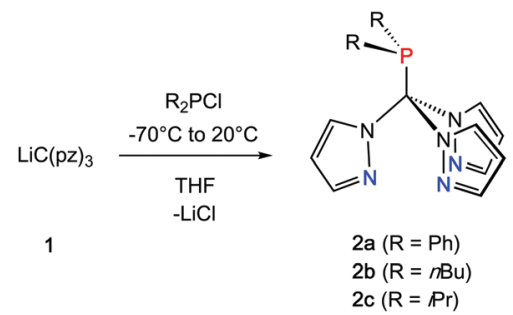

Scheme 1 Synthesis of $2 a-c(R=P h, n B u, i P r)$.

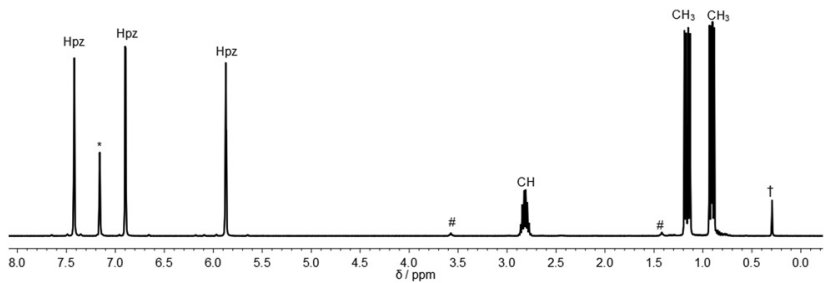

Fig. $1{ }^{1} \mathrm{H}$ NMR spectrum of $2 \mathrm{c}\left(\mathrm{C}_{6} \mathrm{D}_{6}\left(^{*}\right), 300 \mathrm{MHz}, \#=\mathrm{THF}, \dagger=\right.$ grease). ducts 2a-c were obtained in analytically pure form in $65-71 \%$ isolated yield (Scheme 1).

The ${ }^{1} \mathrm{H}$ NMR spectra of the three ligands in benzene- $d_{6}$ show the expected equivalence of all three pyrazolyl rings within one molecule. While the resonances of the phenyl groups in 2a appear in typical multiplets with a chemical shift of $\delta=7.03 \mathrm{ppm}$ ( $\mathrm{m}$-, $p$-position) and $7.98 \mathrm{ppm}$ ( $o$-position), 2b and $2 \mathrm{c}$ feature diastereotopic protons for the $n \mathrm{Bu}$ and iPr substituents, as exemplified for $2 \mathrm{c}$ in Fig. 1 (see also ESI $\$$ ). Due to ${ }^{1} J_{\mathrm{P}, \mathrm{C}}$ couplings, the carbon atoms in apical position of the tris(pyrazolyl)methyl entities in 2a-c appear as doublets with chemical shifts between $\delta=95.2$ and $96.0 \mathrm{ppm}$ and coupling constants of 44.1-55.8 Hz.

As expected for alkyl and aryl phosphines, the ${ }^{31} \mathrm{P}\left\{{ }^{1} \mathrm{H}\right\}$ NMR appear at $\delta=37.0 \mathrm{ppm}$ for $\mathbf{2 b}, \delta=47.2 \mathrm{ppm}$ for $\mathbf{2 a}$ and $\delta=$ 59.9 ppm for $2 \mathrm{c}$, respectively. ${ }^{14}$

In order to unambiguously confirm the formation of the products, X-ray structure analyses were conducted on single crystals of 2a-c (Fig. 2). The compounds crystallise in the triclinic, monoclinic and orthorhombic space groups $P \overline{1}$ (2b), $P 2_{1} / c$ (2c) and $P n a 2_{1}$ (2a), respectively (ESI + ). Relevant structural data are listed in the caption of Fig. 2. As already anticipated from the NMR data, the apical carbon atom of the $\mathrm{C}(\mathrm{pz})_{3}$ entities are in bound to the $\mathrm{PR}_{2}$ donor sets. In each case, the three pyrazolyl substituents adopt a "propeller"-like arrangement with the additional nitrogen donor atoms $\left(\mathrm{N}_{\mathrm{pz}}\right)$ pointing in direction of the $\mathrm{PR}_{2}$ group. Similar effects have been observed before for backbone-functionalised tris(pyrazolyl)silanides such as $\mathrm{R}_{3} \mathrm{Si}-\mathrm{Si}\left(3,5-\mathrm{Me}_{2} \mathrm{pz}\right)_{3}$ or $\mathrm{R}_{3} \mathrm{Sn}-\mathrm{Si}\left(3,5-\mathrm{Me}_{2} \mathrm{pz}\right)_{3}{ }^{5 a}$ Although these structural aspects presumably can be attributed to steric repulsion between the individual pyrazolyl heterocycles, we cannot exclude additional $\mathrm{P} \cdots \mathrm{N}_{\mathrm{pz}}$ interactions in these compounds. In all three cases, however, The P1-C1 bond lengths of $194.6 \mathrm{pm}(\mathbf{2 a}), 192.2 \mathrm{pm}$ (2b) and $192.7 \mathrm{pm}$ (2c) are slightly longer than regular C-P single bonds (ca. $184 \mathrm{pm})$. It is worth mentioning that these

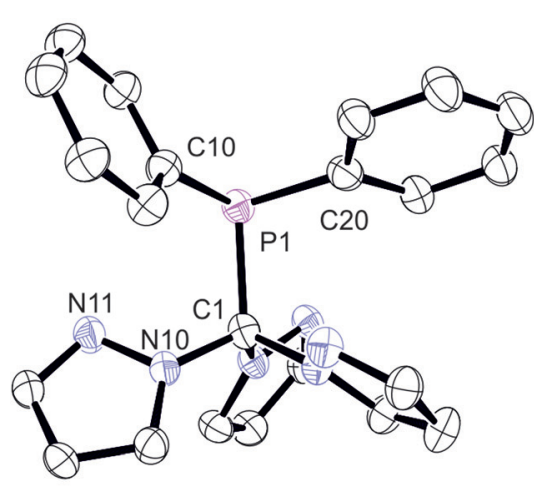

2a

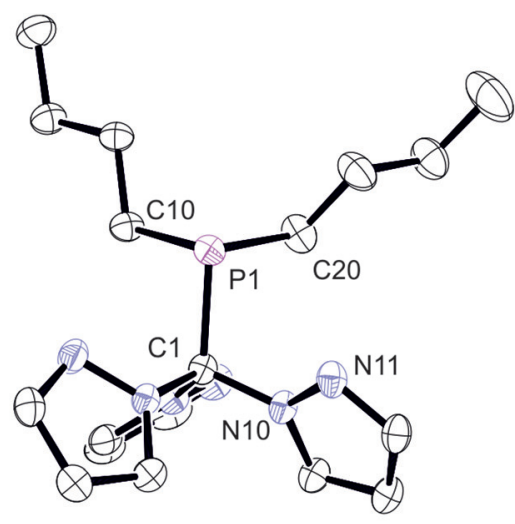

$2 b$

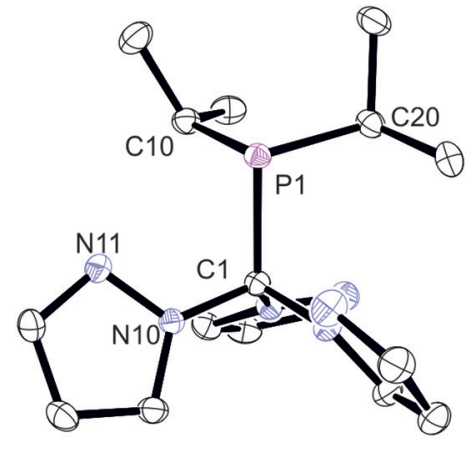

2c

Fig. 2 Molecular structures of the ligands $2 \mathrm{a}-\mathrm{c}$. The thermal ellipsoids are at $30 \%$ probability level. Hydrogen atoms have been omitted for clarity. Selected bond lengths [pm] and angles [०]: (2a) C1-P1 194.6(5), C1-N10 145.4(6), C1-N20 146.4(6), C1-N30 144.2(6) P1-C10 182.8(5), P1-C20 183.1(6), P1-C1-N10 115.0(3), P1-C1-N20 107.0(3), P1-C1-N30 111.3(3). (2b) C1-P1 192.2(13), C1-N10 145.3(16), C1-N20 146.1(14), C1-N30 145.1(16) P1-C10 184.8(16), P1-C20 184.2(16), P1-C1-N10 108.5(8), P1-C1-N20 114.6(9), P1-C1-N30 111.3(9). (2c) C1-P1 192.7(14), C1-N10 145.5(18), C1-N20 145.7(17), C1-N30 146.5(16) P1-C10 186.4(15), P1-C20 187.2(16), P1-C1-N10 109.4(9), P1-C1-N20 109.1(9), P1-C1-N30 115.2(9). 
are the first phosphine-functionalised Tpm derivatives featuring additional $\mathrm{C}_{\text {apical }}$-bound $\mathrm{PR}_{2}$ donor sets.

\section{Rhodium carbonyl complexes of $2 \mathrm{a}-\mathrm{c}$}

In order to shed some light on the coordination behaviour of this novel class of ligands, we investigated various d-block metal complexes. The mononuclear rhodium carbonyl complexes 3a-c were synthesised by treating the precursor $\left[\mathrm{Rh}(\mathrm{CO})_{2} \mathrm{Cl}\right]_{2}$ with $\mathbf{2 a - c}$ (Scheme 2$)$. Analytically pure crystalline solids were obtained in very good yields of $80-87 \%$. The microanalytical and IR spectral data provided evidence that only one carbonyl ligand is present in the complexes and that the overall composition should be $[(2 \mathbf{a}-\mathbf{c}) \mathrm{Rh}(\mathrm{CO}) \mathrm{Cl}]$ with the hybrid ligands either acting as bi- or tridentate chelate. In the ${ }^{1} \mathrm{H}$ NMR spectra, only three equivalent pyrazolyl rings are visible, which is most likely caused by a rapid exchange in solution. This phenomenon has already been observed for Tpms complexes (Chart 1), where the presence of a $C_{3}$ symmetric $\kappa^{3} N$-coordinated species alongside the heterochelate complexes could be verified in solution. ${ }^{15}$ In our case, however, the ${ }^{31} \mathrm{P}\left\{{ }^{1} \mathrm{H}\right\}$ spectra contradict the presence of a $\kappa^{3} N$ coordinated species. Besides the coupling to the rhodium nucleus, the signals of the phosphorous atoms in the ${ }^{31} \mathrm{P}\left\{{ }^{1} \mathrm{H}\right\}$

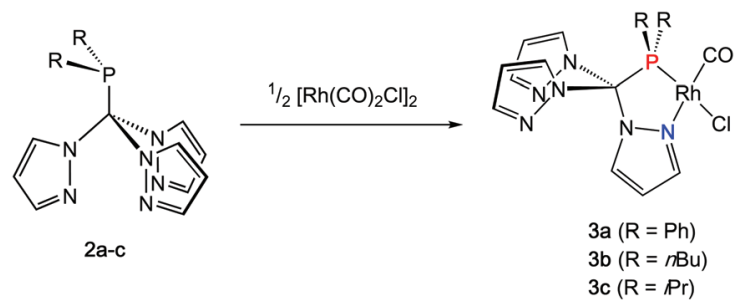

Scheme 2 Synthesis of $3 a-c$
NMR spectra show strong low-field shifts to $\delta=112-144 \mathrm{ppm}$ as compared to the free ligands. This would be in accordance with the assumption of having just a dynamic interchange between the three pz rings in solution and no presence of a $\kappa^{3} N$-coordinated species.

We were able to obtain crystals suitable for X-ray diffraction of all three complexes confirming that the $\mathrm{TpmPR}_{2}$ ligands act as a $\kappa^{2} N, P$-hybrid ligand with two uncoordinated pyrazolyl moieties (Fig. 3, $P 2_{1} / c$ for $3 \mathbf{a}$ and $3 \mathbf{b} ; P 2_{1}$ for $3 \mathbf{c}$, ESI + ). The Rh1-P1 bond lengths range between 219-222 pm, whereas the Rh1-N11 bond lengths show values between 206 and 208 pm. The $\mathrm{P} 1-\mathrm{C} 1$ bond lengths are in the same range as observed for the uncoordinated ligands.

The IR spectra in solid state were recorded for compounds 3a-c and compared to the TEP values of the corresponding phosphines in $\mathrm{CH}_{2} \mathrm{Cl}_{2}$ solution (Table 1). ${ }^{16}$ As expected, the same tendencies in the donor capacities observed for the "classical" aryl- or alkyl phosphines can also be observed for the tris(pyrazolyl)methyl phosphines ( $\mathrm{Ph}<n \mathrm{Bu}<\mathrm{iPr})$.

\section{Coordination flexibility}

As already mentioned before, different coordination modes are known from complexes containing Tpms as ligand. In Tpms, the oxygen atoms are very weak donor atoms and as a result, dynamic equilibria are observed in solution. We also became

Table 1 Wave numbers of compounds $3 a-c$ in comparison to the TEP values of their corresponding phosphines ${ }^{16}$

\begin{tabular}{llll}
\hline Ligand & $\nu(\mathrm{CO})\left[\mathrm{cm}^{-1}\right]$ & Ligand & TEP $\left[\mathrm{cm}^{-1}\right]$ \\
\hline 3a & 1994 & $\mathrm{PPh}_{3}$ & 2069 \\
3b & 1981 & $\mathrm{PnBu}_{3}$ & 2060 \\
3c & 1978 & $\mathrm{PiPr}_{3}$ & 2059
\end{tabular}

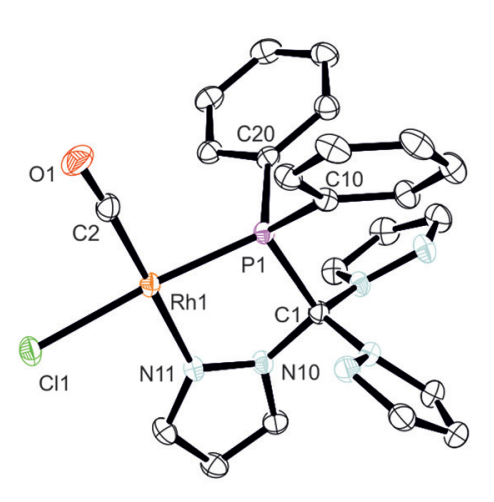

$3 a$

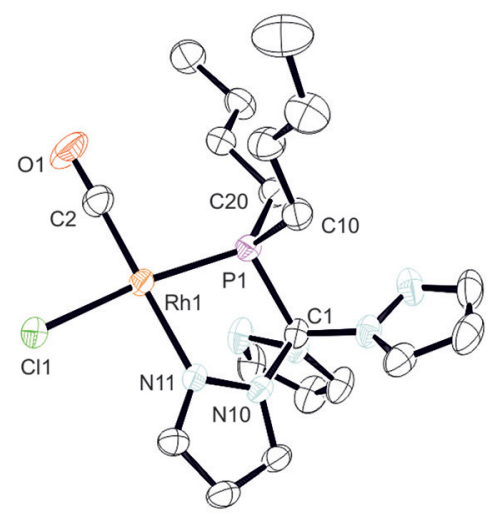

$3 b$

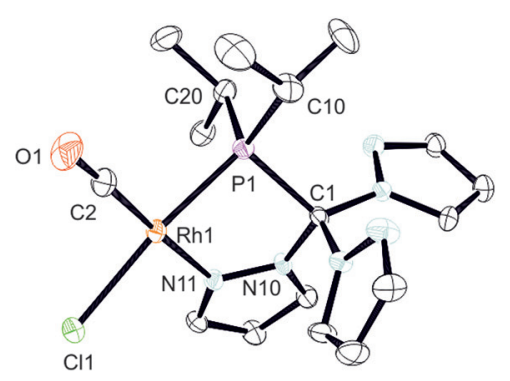

$3 c$

Fig. 3 Molecular structures of complexes 3a-c. The thermal ellipsoids are at 30\% probability level. Hydrogen atoms have been omitted for clarity. Selected bond lengths [pm] and angles [ ${ }^{\circ}$ ]: (3a) Rh1-P1 221.16(7), Rh1-Cl1 237.7(7), Rh1-N11 206.34(17), Rh1-C2 182.0(2), P1-C1 195.56(19), P1Rh1-Cl1 174.195(19), N11-Rh1-P1 83.78(5), N11-Rh1-Cl1 90.44(6), C2-Rh1-P1 97.02(7), C2-Rh1-Cl1 88.76(7), C2-Rh1-N11 178.90(8). (3b) Rh1P1 219.14(13), Rh1-Cl1 235.50(14), Rh1-N11 208.6(3), Rh1-C2 180.1(5), P1-C1 192.4(4), P1-Rh1-Cl1 168.96(5), N11-Rh1-P1 81.67(10), N11-Rh1-Cl1 89.81(10), C2-Rh1-P1 97.12(17), C2-Rh1-Cl1 91.59(16), C2-Rh1-N11 178.03(17). (3c) Rh1-P1 221.84(11), Rh1-Cl1 235.88(13), Rh1-N11 207.6(3), Rh1-C2 180.4(5), P1-C1 195.0(4), P1-Rh1-Cl1 169.85(4), N11-Rh1-P1 81.48(12), N11-Rh1-Cl1 88.39(12), C2-Rh1-P1 96.81(19), C2-Rh1-Cl1 93.30(19), C2-Rh1-N11 177.8(3). 
interested to studying the coordination flexibility of our new hybrid ligands $\mathbf{2 a - c}$ and, thus, investigated the coordination chemistry of $\mathbf{2 a}$ or $\mathbf{2 c}$ as representatives for the new ligand family in more detail.

Since $\left[\mathrm{CpRu}(\mathrm{acn})_{3}\right]\left[\mathrm{PF}_{6}\right](\mathrm{acn}=$ acetonitrile $)$ is a valuable precursor for transferring a $\{\mathrm{RuCp}\}$ entity to tripodal ligands such as Tpm or Tpmd, ${ }^{4 n, 17}$ we inspected its reactivity towards $2 \mathrm{c}$ (Scheme 3). Orange crystals of 4 were isolated in $91 \%$ yield. ${ }^{1} \mathrm{H}$ NMR spectroscopic investigations revealed that the hybrid ligand is coordinated in a $\kappa^{3} N, N^{\prime}, P$ fashion. The detection of one uncoordinated pyrazolyl ring indicated no interchange of the pz entities in solution in this case. The down field shift of the phosphorous signal in the ${ }^{31} \mathrm{P}\left\{{ }^{1} \mathrm{H}\right\}$ NMR spectrum of 4 is even stronger than for $\mathbf{3 a}-\mathbf{c}(\delta=170.4 \mathrm{ppm})$. The molecular structure was established confirming the $\kappa^{3} N, N, P$-hetero-

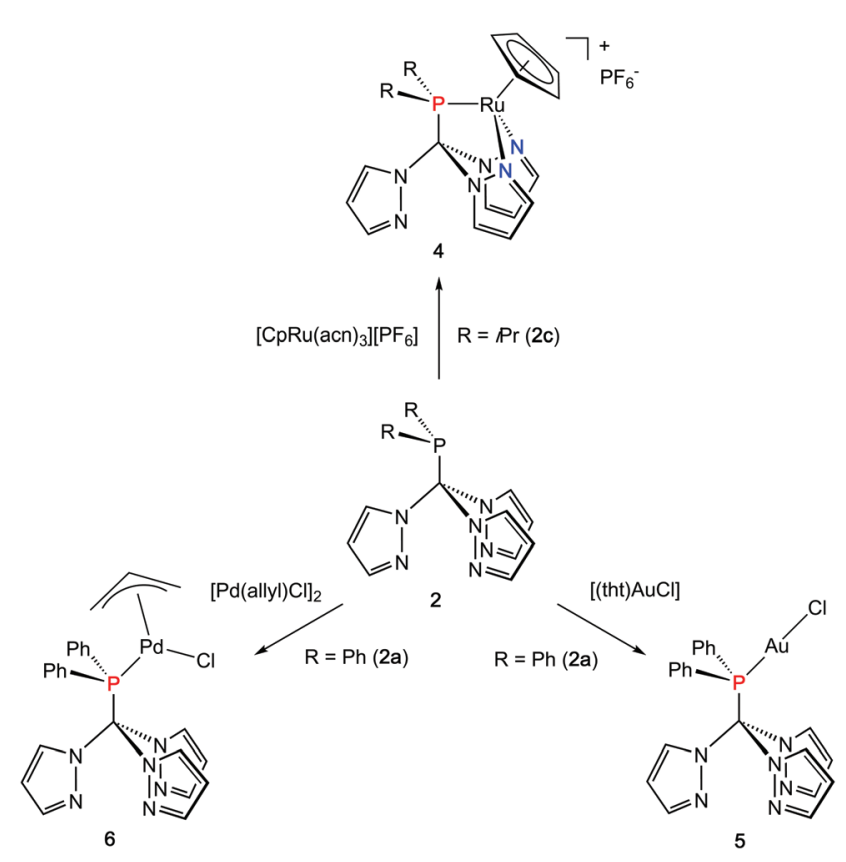

Scheme 3 Synthesis of 4-6. scorpionate structure (Fig. 4, space group Pbcn, ESI coordination sphere of the ruthenium atom is complemented by one $\eta^{5}-\mathrm{C}_{5} \mathrm{H}_{5}$ ligand. The average Ru1-N bond length in 4 was found to amount to $209.1 \mathrm{pm}$, which is only slightly shorter than in comparable $\kappa^{3} N$-Tpm $(211.1 \mathrm{pm})^{17}$ and $\kappa^{3} N$ Tpmd $(210.6 \mathrm{pm})^{4 n}$ complexes published by our group. The Ru1-P1 bond length of 229.3 pm is in good agreement with those observed for other complexes from the literature. ${ }^{18}$

Another metal precursor which was reacted with 2 a was [Au(tht)Cl] (tht = tetrahydrothiophene) and complex 5 was obtained in $82 \%$ isolated yield as colourless crystalline solid. Considering that $\mathrm{Au}(\mathrm{I})$ is a rather soft metal ion and prefers linear coordination, we expected the $\mathrm{P}$ donor in 2a to solely act as 2-electron donor. Indeed, the NMR spectroscopic investigations reveal just one set of signals concerning the pyrazolyl protons. The coordination of the gold atom to the $\mathrm{P}$ donor causes a low field shift of the phosphorous atom in the ${ }^{31} \mathrm{P}$ $\left\{{ }^{1} \mathrm{H}\right\}$ NMR spectra from $\delta=47.2 \mathrm{ppm}$ in $2 \mathrm{a}$ to $\delta=78.7 \mathrm{ppm}$ in 5. The molecular structure depicted in Fig. 4 (space group $P 2_{1} / n$ ) illustrates the almost linear coordination of the gold atom $\left(\mathrm{P} 1-\mathrm{Au} 1-\mathrm{Cl} 1=175.5^{\circ}\right)$. Other structural parameters remain almost unchanged compared to the uncoordinated ligand.

As a representative of group 10 metals, 2a was reacted with $[\mathrm{Pd}(\text { allyl }) \mathrm{Cl}]_{2}$ to provide a hardly soluble, pale yellow complex 6, which was isolated in rather low yields of $48 \%$ in an analytical pure form. Like in $\mathbf{5}$, the phosphorous atom is acting as donor ligand whereas the pyrazolyl rings stay uncoordinated. The downfield shift of the ${ }^{31} \mathrm{P}\left\{{ }^{1} \mathrm{H}\right\}$ chemical shift of $\delta=$ 89.4 ppm is even more distinct than for $\mathbf{5}$.

Crystals suitable for X-ray crystallography could be obtained from a THF/hexane mixture (Fig. 4, space group $P 22_{1} 2_{1} 2_{1}$, ESI + ). The Pd1-P1 bond length is $232.3 \mathrm{pm}$ and in accordance to similar Pd-P bonds known from literature. ${ }^{18}$

\section{Heterobimetallic complex of 2 a}

In an effort to prepare heterobimetallic complexes of the new hybrid ligands, various attempts have been made by treating 4-6 with various other metal sources, all of which were

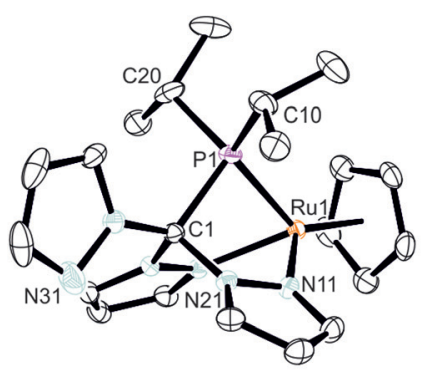

4

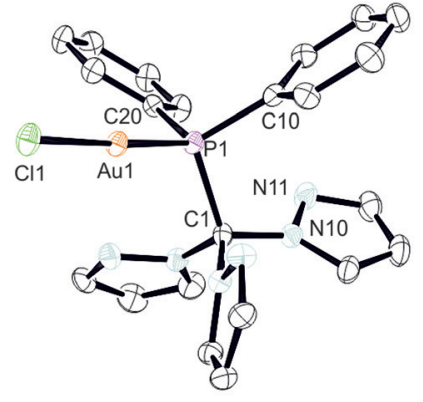

5

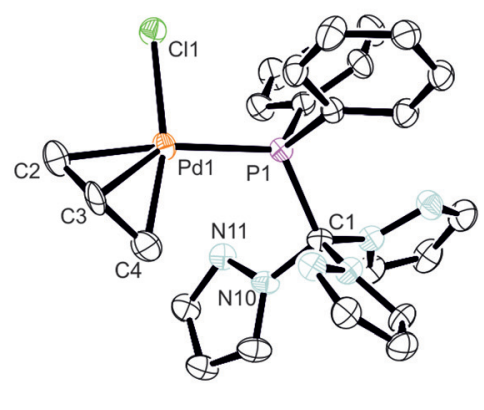

6

Fig. 4 Molecular structures of 4-6. The thermal ellipsoids are at 30\% probability level. Hydrogen atoms and the anion (4) have been omitted for clarity. Selected bond lengths [pm] and angles []: 4: Ru1-P1 229.27(6), Ru1-N11 209.26(18), Ru1-N21 209.00(17), Ru1-Cp cent 181.9 P1-C1 193.9(2), $\mathrm{P} 1-\mathrm{Ru1}-\mathrm{Cp} \mathrm{p}_{\text {cent }}$ 135.27, N11-Ru1-Cp cent 145.60, N21-Ru1-Cp cent 130.00, N11-Ru1-P1 78.93(5), N21-Ru1-P1 82.74(7); 5: Au1-P1 = 223.38(15), Au1-Cl1 = 227.66(15), C1-P1 = 194.2(6), P1-Au1-Cl1 176.46(5), C1-P1-Au1 = 111.54(18), Au1-P1-C200 = 114.93(19); 6: Pd1-P1 232.0(2), Pd1-Cl1 235.4(2), P1-C1 196.0(9), P1-Pd1-Cl1 93.97(8), Pd1-P1-C1 116.5(3). ( $C p_{\text {cent }}=$ centroid of the Cp ligand). 


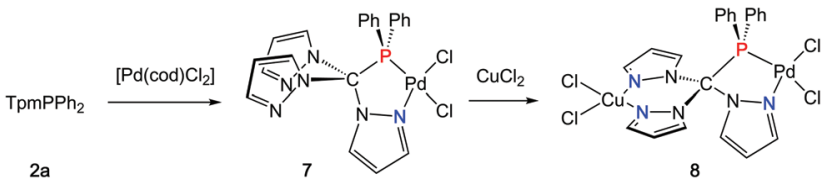

Scheme 4 Synthesis of 7 and 8.

not successful. However, sequential treatment of 2a with $\left[\mathrm{Pd}(\operatorname{cod}) \mathrm{Cl}_{2}\right]$ and $\mathrm{CuCl}_{2}$ provided the suitable entry point to the first heterobimetallic complex 8 (Scheme 4).

The initial product ( 7 ) of this reaction is a yellow solid and was obtained in very good yields (87\%). As evidenced by ${ }^{1} \mathrm{H}$ and ${ }^{31} \mathrm{P}$ NMR ( $\left.\delta=90.7 \mathrm{ppm}\right)$ spectroscopic investigations, the palladium atom is coordinated in a $\kappa^{2} P, N$-coordination mode leaving two pyrazolyl moieties uncoordinated. The palladium atom in 7 is coordinated in a square planar coordination mode (Fig. 5, space group $P 2_{1} / c$, ESI $\$$ ). Due to the different trans-influence of phosphorous and nitrogen donors, the
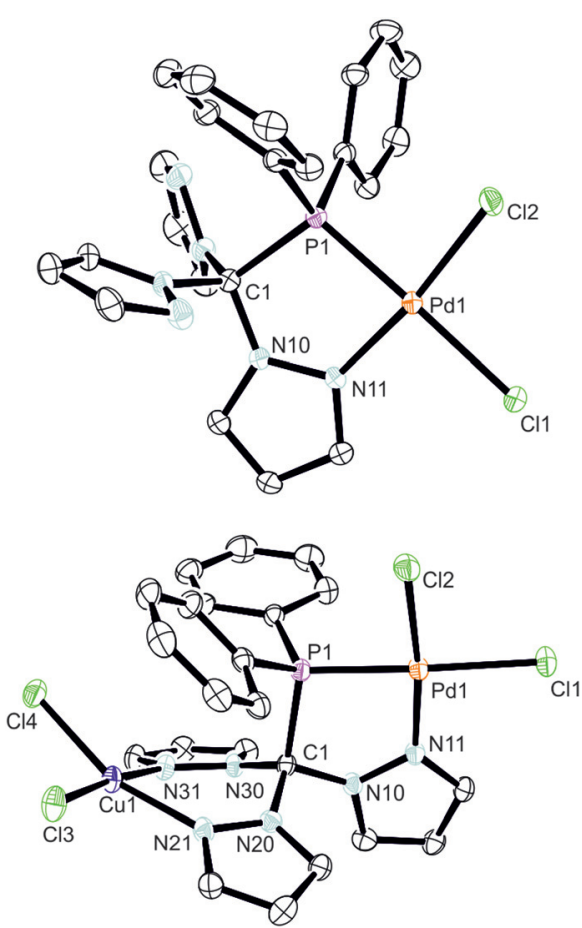

Fig. 5 Molecular structures of 7 and 8 . The thermal ellipsoids are at $30 \%$ probability level. Hydrogen atoms and solvent molecules (8: acetonitrile) have been omitted for clarity. Selected bond lengths [pm] and angles [*]: 7: $\mathrm{Pd} 1-\mathrm{P} 1=218.3(6), \mathrm{Pd} 1-\mathrm{Cl} 1=237.0(6), \mathrm{Pd} 1-\mathrm{Cl} 2=$ 228.42(6), $\mathrm{Pd} 1-\mathrm{N} 11=201.41(16), \mathrm{P} 1-\mathrm{C} 1=196.2(2), \mathrm{P} 1-\mathrm{Pd} 1-\mathrm{Cl}$ 175.411(19), P1-Pd1-Cl2 87.87(2), Cl2-Pd1-Cl1 = 94.91(2), N11-Pd1$\mathrm{P} 1=84.8, \mathrm{~N} 11-\mathrm{Pd} 1-\mathrm{Cl} 1$ 92.31(5), N11-Pd1-172.22(5). 8: Pd1-P1 219.56(17), Pd1-Cl1 236.21(17), Pd1-Cl2 227.67(18), Pd1-N11 200.5(5), Ci1-Cl3 221.46(19), Cu1-N21 202.6(5), Cu1-N31 201.4(5), P1-C1 195.2(6), Pd1‥Cu1 603.61(14), P1-Pd1-Cl1 172.30(6), P1-Pd1-Cl2 87.97(7), Cl2-Pd1-Cl1 94.47(7), N11-Pd1-P1 84.84(15), N11-Pd1-Cl1 92.67(15), N11-Pd1-Cl2 172.81(15), Cl3-Cu1-Cl4 94.63(8), N21-Cu1$\mathrm{Cl} 3$ 91.32(15), N21-Cu1-Cl4 161.49(15), N31-Cu1-N21 84.8(2), N31-Cu1-Cl4 90.07(15), N31-Cu1-Cl3 174.86(15).
Pd1-Cl1 bond length is slightly longer $(237.0 \mathrm{pm})$ than Pd1-Cl2 (228.4 pm). The Pd1-P1 bond (218.3 pm) is remarkably shorter than the Pd1-P1 bond in complex 6 (232.3 pm).

By reacting 7 with $\mathrm{CuCl}_{2}$, the first heterobimetallic complex 8 was successfully isolated and characterised (Fig. 5, space group $P 2_{1} / c$, ESI $\$$ ). The coordination sphere of the palladium atom is not affected by the coordination of $\mathrm{Cu}$ (II) since all bond lengths and angles stay almost the same. The average $\mathrm{Cu}-\mathrm{N}$ and $\mathrm{Cu}-\mathrm{Cl}$ bond lengths were found to amount to 223.6 pm and $202.1 \mathrm{pm}$, respectively. The square planar coordination mode at the copper atom is rather distorted as judged from the N21-Cu1-Cl4 angle of only 161.5(2) degrees. This distortion is also manifested in the boat-shaped arrangement of the six-membered chelate ring, which, however, has already been described for related copper complexes in the literature. $^{19}$

\section{Conclusions}

In summary, we have introduced a novel class of phosphinefunctionalised Tpm ligands (2a-c), which proved to be a very flexible class of hybrid ligands due to many different coordination modes observed for several transition metal complexes (3-7). Furthermore, we were able to synthesise the first heterobimetallic complex of a tris(pyrazolyl)methyl phosphine containing the metals palladium and copper. Further investigations regarding the cooperativity along the two metals within this complex and the synthesis of even more multimetallic complexes are part of ongoing research in our group.

\section{Experimental section}

\section{General methods and materials}

All manipulations were carried out with standard Schlenk line and dry-box techniques in a dry argon atmosphere. Methylene chloride and acetonitrile were freshly distilled in an argon atmosphere from calcium hydride. Toluene, diethyl ether and tetrahydrofuran were dried using sodium/benzophenone ketyl. $\mathrm{CD}_{2} \mathrm{Cl}_{2}$ was vacuum transferred from calcium hydride while $\mathrm{C}_{6} \mathrm{D}_{6}$ and thf- $d 8$ were vacuum transferred from sodium/benzophenone ketyl into thoroughly dried glassware equipped with Young Teflon valves.

$\mathrm{Tpm}^{9 b}$ and $n \mathrm{Bu}_{2} \mathrm{PCl}^{20}$ were synthesized in accordance to the literature. All other reagents were purchased from Aldrich or Roth and used without further purification.

${ }^{1} \mathrm{H},{ }^{13} \mathrm{C}$, spectra were recorded with Bruker AV 300 and 400 spectrometers in dried deuterated solvents. The chemical shifts are expressed in parts per millions and ${ }^{1} \mathrm{H}$ and ${ }^{13} \mathrm{C}$ signals are given relative to TMS. Coupling constants $J$ are given in hertz as positive values regardless of their real individual signs. The multiplicity of the signals is indicated as s, d, q, sept or $\mathrm{m}$ for singlets, doublets, quartets, septets or multiplets, respectively. The assignments were confirmed as necessary with the use of 2D NMR correlation experiments. Mass spec- 
trometry measurements were performed on an Advion expression $^{\mathrm{L}}$ CMS mass spectrometer under atomic pressure chemical ionization (APCI) or under electrospray ionization (ESI). IR spectra were measured with a Bruker Alpha spectrometer using the attenuated total reflection (ATR) technique on powdered samples, and the data are quoted in wavenumbers $\left(\mathrm{cm}^{-1}\right)$. The intensity of the absorption band is indicated as vw (very weak), w (weak), $\mathrm{m}$ (medium), $\mathrm{s}$ (strong), vs. (very strong) and br (broad). Melting points were measured with a Thermo Fischer melting point apparatus and are not corrected. Elemental analyses were carried out in the institutional technical laboratories of the Karlsruhe Institute of Technology (KIT).

General synthesis of $\mathrm{TpmPR}_{2}$ with $\mathrm{R}=\mathbf{P h}(2 \mathrm{a}), n \mathrm{Bu}(2 \mathrm{~b})$ and iPr (2c). A solution of tris(pyrazolyl)methane $(16 \mathrm{mmol}$, 1 equiv.) in THF is cooled down to $-78{ }^{\circ} \mathrm{C}$ and $10 \mathrm{~mL}$ of a $1.6 \mathrm{M}$ solution of $n \mathrm{BuLi}$ ( $16 \mathrm{mmol}, 1$ equiv.) are added dropwise. The white suspension is stirred for another hour at $-78{ }^{\circ} \mathrm{C}$, then $\mathrm{R}_{2} \mathrm{PCl}$ ( 16 mmol, 1 equiv.) is added slowly. The resolving brown reaction mixture is stirred over night at RT. The solvents are removed in vacuo and the residue is dissolved in toluene. The insoluble solid is removed by filtration. A moisture and air sensitive colourless product is obtained by concentrating the brown solution under reduced pressure. Crystals are obtained by slow diffusion of a THF solution in hexane $(1: 2)$.

Data for $2 \mathrm{a}(\mathbf{R}=\mathbf{P h})$. Colourless solid, yield: $70 \% .{ }^{1} \mathrm{H}$ NMR $\left(300.13 \mathrm{MHz}, \mathrm{C}_{6} \mathrm{D}_{6}\right): \delta=5.75\left(\mathrm{ddd},{ }^{3} J_{\mathrm{HH}}=2.6 \mathrm{~Hz},{ }^{3} J_{\mathrm{HH}}=1.9 \mathrm{~Hz}\right.$, $\left.{ }^{5} J_{\mathrm{HP}}=0.6 \mathrm{~Hz}, 3 \mathrm{H}, 4-H_{\mathrm{pz}}\right), 6.62\left(\mathrm{dd},{ }^{3} J_{\mathrm{HH}}=2.6 \mathrm{~Hz},{ }^{4} J_{\mathrm{HH}}=0.6 \mathrm{~Hz}\right.$, $\left.3 \mathrm{H}, 5-H_{\mathrm{pz}}\right), 7.03\left(\mathrm{~m}, 6 \mathrm{H}, m, p-H_{\mathrm{Ar}}\right), 7.31\left(\mathrm{dd},{ }^{3} J_{\mathrm{HH}}=1.7 \mathrm{~Hz},{ }^{4} J_{\mathrm{HH}}\right.$ $\left.=0.6 \mathrm{~Hz}, 3 \mathrm{H}, 3-H_{\mathrm{pz}}\right), 7.98\left(\mathrm{~m}, 4 \mathrm{H}, o-H_{\mathrm{Ar}}\right) \mathrm{ppm} \cdot{ }^{13} \mathrm{C}\left\{{ }^{1} \mathrm{H}\right\} \mathrm{NMR}$ $\left(75.48 \mathrm{MHz}, \mathrm{C}_{6} \mathrm{D}_{6}\right): \delta=96.0\left(\mathrm{~d},{ }^{1} J_{\mathrm{CP}}=48.1 \mathrm{~Hz}, C_{\text {apical }}\right), 106.3(\mathrm{~d}$, $\left.{ }^{4} J_{\mathrm{CP}}=1.6 \mathrm{~Hz}, 4-C_{\mathrm{pz}}\right), 127.8\left(\mathrm{~d},{ }^{3} J_{\mathrm{CP}}=7.2 \mathrm{~Hz}, m-C_{\mathrm{Ar}}\right), 128.9(\mathrm{~s}$, $\left.p-C_{\mathrm{Ar}}\right), 130.3\left(\mathrm{~s}, 5-C_{\mathrm{pz}}\right), 135.7\left(\mathrm{~d},{ }^{2} J_{\mathrm{CP}}=22.4 \mathrm{~Hz}, o-C_{\mathrm{Ar}}\right), 135.9(\mathrm{~d}$, ${ }^{1} J_{\mathrm{CP}}=21.5 \mathrm{~Hz}$, ipso- $\left.C_{\mathrm{Ar}}\right), 141.0\left(\mathrm{~s}, 3-C_{\mathrm{pz}}\right) \mathrm{ppm} .{ }^{31} \mathrm{P}\left\{{ }^{1} \mathrm{H}\right\} \mathrm{NMR}$ (121.49 MHz, $\mathrm{C}_{6} \mathrm{D}_{6}$ ): $\delta=47.2$ (s) ppm. EA [\%] calc. $\mathrm{C}_{22} \mathrm{H}_{19} \mathrm{~N}_{6} \mathrm{P}$ : C 66.32, H 4.81, N 21.09; found C 66.65, H 4.81, N 20.20. APCI-MS $\left(\mathrm{m} / \mathrm{z}\right.$ ): calc. 398.14 , found $399.3[\mathrm{M}+\mathrm{H}]^{+}$. FT-IR (solid, ATR, $\mathrm{cm}^{-1}$ ): 3137 (vw), 3118 (vw), 3054 (vw), 1585 (vw), 1507 (vw), 1476 (vw), 1433 (vw), 1417 (w), 1384 (m), 1323(w), $1311(\mathrm{w}), 1242(\mathrm{w}), 1199(\mathrm{w}), 1157$ (vw), 1095 (m), $1083(\mathrm{~m})$, 1061 (w), 1043 (w), 1028 (vw), 979 (vw), 915 (vw), $879(\mathrm{w})$, $842(\mathrm{~s}), 770(\mathrm{w}), 739(\mathrm{vs}), 694(\mathrm{~s}), 659(\mathrm{w}), 605(\mathrm{~m}), 503(\mathrm{~m})$, $486(\mathrm{~m}), 449(\mathrm{~m}), 412(\mathrm{w}), 382(\mathrm{w})$. Mp (sealed tube under Ar): 135.8-137.2 ${ }^{\circ} \mathrm{C}$.

Data for $2 \mathbf{b}(\mathbf{R}=\boldsymbol{n} \mathbf{B u})$. Colourless solid, yield: $71 \% .{ }^{1} \mathrm{H}$ NMR $\left(300.13 \mathrm{MHz}, \mathrm{C}_{6} \mathrm{D}_{6}\right): \delta=0.84\left(\mathrm{t},{ }^{3} J_{\mathrm{HH}}=7.3 \mathrm{~Hz}, 6 \mathrm{H}, \mathrm{CH}_{3}\right), 1.38$ (m, $4 \mathrm{H}, \mathrm{CH}_{2}-\mathrm{Me}$ ), $1.59\left(\mathrm{~m}, 4 \mathrm{H}, \mathrm{CH}_{2}-\mathrm{Et}\right), 1.87$ (dddd, ${ }^{2} J_{\mathrm{HP}}=$ $4.5 \mathrm{~Hz},{ }^{2} J_{\mathrm{HH}}=13.6 \mathrm{~Hz},{ }^{3} J_{\mathrm{HH}}=10.0 \mathrm{~Hz},{ }^{3} J_{\mathrm{HH}}=5.7 \mathrm{~Hz}, 2 \mathrm{H}$, $\left.\mathrm{CH}_{a} H_{b}-\mathrm{Pr}\right), 2.22\left(\mathrm{ddd},{ }^{2} J_{\mathrm{HP}}=13.6 \mathrm{~Hz},{ }^{3} J_{\mathrm{HH}}=10.0 \mathrm{~Hz},{ }^{3} J_{\mathrm{HH}}=\right.$ $5.7 \mathrm{~Hz}, 2 \mathrm{H}, \mathrm{CH}_{a} H_{b}-\mathrm{Pr}$ ), $5.86\left(\mathrm{ddd},{ }^{3} J_{\mathrm{HH}}=2.6 \mathrm{~Hz},{ }^{3} J_{\mathrm{HH}}=1.7 \mathrm{~Hz}\right.$, $\left.{ }^{5} J_{\mathrm{HP}}=0.6 \mathrm{~Hz}, 3 \mathrm{H}, 4-H_{\mathrm{pz}}\right), 6.63\left(\mathrm{dd},{ }^{3} J_{\mathrm{HH}}=2.6 \mathrm{~Hz},{ }^{3} J_{\mathrm{HH}}=0.8 \mathrm{~Hz}\right.$, $\left.3 \mathrm{H}, 5-H_{\mathrm{pz}}\right), 7.48\left(\mathrm{dd},{ }^{3} J_{\mathrm{HH}}=1.7 \mathrm{~Hz},{ }^{3} J_{\mathrm{HH}}=0.6 \mathrm{~Hz}, 3 \mathrm{H}, 3-H_{\mathrm{pz}}\right)$ ppm. ${ }^{13} \mathrm{C}\left\{{ }^{1} \mathrm{H}\right\}$ NMR $\left(75.48 \mathrm{MHz}, \mathrm{C}_{6} \mathrm{D}_{6}\right): \delta=14.1\left(\mathrm{~s}, \mathrm{CH}_{3}\right), 24.8$ (d, ${ }^{3} J_{\mathrm{CP}}=14.1 \mathrm{~Hz}, \mathrm{CH}_{2}-\mathrm{Me}$ ), 26.7 (d, ${ }^{1} J_{\mathrm{CP}}=20.6 \mathrm{~Hz}, \mathrm{CH}_{2}-\mathrm{Pr}$ ), $29.6\left(\mathrm{~d},{ }^{2} J_{\mathrm{CP}}=24.6 \mathrm{~Hz}, \mathrm{CH}_{2}-\mathrm{Et}\right), 95.4\left(\mathrm{~d},{ }^{1} J_{\mathrm{CP}}=44.1 \mathrm{~Hz}, C_{\text {apical }}\right)$, $106.2\left(\mathrm{~s}, 4-C_{\mathrm{pz}}\right), 130.0\left(\mathrm{~s}, 5-C_{\mathrm{pz}}\right), 141.2\left(\mathrm{~s}, 3-C_{\mathrm{pz}}\right) \mathrm{ppm} .{ }^{31} \mathrm{P}\left\{{ }^{1} \mathrm{H}\right\}$ NMR (121.49 MHz, $\left.\mathrm{C}_{6} \mathrm{D}_{6}\right): \delta=37.0$ (s) ppm. EA [\%] calc.
$\mathrm{C}_{18} \mathrm{H}_{27} \mathrm{~N}_{6} \mathrm{P}: \mathrm{C} 60.32, \mathrm{H} 7.59, \mathrm{~N} 23.45$; found C 60.34, H 7.69, N 23.16. MS (EI, $70 \mathrm{eV}$ ) $\mathrm{m} / \mathrm{z}: 358.2$ (M, 2.8\%), $291.2\left([\mathrm{M}-\mathrm{pz}]^{+}\right.$, 100\%), $213.1\left(\left[{ }^{\mathrm{H}} \mathrm{Tpmd}\right]^{+}, 97.9 \%\right), 147.1\left(\left[{ }^{\mathrm{H}} \mathrm{Tpmd}-\mathrm{pz}\right]^{+}, 98.3 \%\right)$ and $146.1\left(\left[\mathrm{HPBu}_{2}\right]^{+}, 15.1 \%\right)$. FT-IR (solid, ATR, $\left.\mathrm{cm}^{-1}\right)$ : $3117(\mathrm{vw}), 2957(\mathrm{w}), 2924(\mathrm{w}), 2858(\mathrm{w}), 1607(\mathrm{vw}), 1507(\mathrm{w})$, $1463(\mathrm{~m}), 1417$ (s), 1385 (s), 1316 (m), 1242 (m), 1195 (m), $1098(\mathrm{~s}), 1086(\mathrm{~m}), 1060(\mathrm{~m}), 1046(\mathrm{~m}), 973(\mathrm{w}), 914(\mathrm{w})$, $896(\mathrm{w}), 844(\mathrm{~s}), 757$ (s), $613(\mathrm{~m}), 454(\mathrm{w}), 424(\mathrm{~m})$. Mp (sealed tube under Ar): $63.4-66.8^{\circ} \mathrm{C}$.

Data for $2 \mathbf{c}(\mathbf{R}=\mathbf{i P r})$. Colourless solid, yield: $65 \% .{ }^{1} \mathrm{H}$ NMR $\left(300.13 \mathrm{MHz}, \mathrm{C}_{6} \mathrm{D}_{6}\right): \delta=0.91\left(\mathrm{dd},{ }^{3} J_{\mathrm{HP}}=12.3 \mathrm{~Hz},{ }^{3} J_{\mathrm{HH}}=7.0 \mathrm{~Hz}\right.$, $\left.6 \mathrm{H}, \mathrm{CH}-\left(\mathrm{CH}_{3}\right)_{2}\right), 1.16\left(\mathrm{dd},{ }^{3} J_{\mathrm{HP}}=15.7 \mathrm{~Hz},{ }^{3} J_{\mathrm{HH}}=7.3 \mathrm{~Hz}, 6 \mathrm{H}\right.$, $\left.\mathrm{CH}-\left(\mathrm{CH}_{3}\right)_{2}\right), 2.82\left(\mathrm{dsep},{ }^{2} \mathrm{HP}_{\mathrm{HP}}=12.5 \mathrm{~Hz},{ }^{3} J_{\mathrm{HH}}=5.2 \mathrm{~Hz}, 2 \mathrm{H}, \mathrm{CH}-\right.$ $\left.\left(\mathrm{CH}_{3}\right)_{2}\right), 5.88\left(\mathrm{ddd},{ }^{3} \mathrm{~J}_{\mathrm{HH}}=2.6 \mathrm{~Hz},{ }^{3} J_{\mathrm{HH}}=1.8 \mathrm{~Hz},{ }^{5} J_{\mathrm{HP}}=2.6 \mathrm{~Hz}\right.$, $\left.3 \mathrm{H}, 4-H_{\mathrm{pz}}\right), 6.90\left(\mathrm{dd},{ }^{3} J_{\mathrm{HH}}=2.6 \mathrm{~Hz},{ }^{3} J_{\mathrm{HH}}=0.7 \mathrm{~Hz}, 3 \mathrm{H}, 5-H_{\mathrm{pz}}\right)$, $7.42\left(\mathrm{dd},{ }^{3} J_{\mathrm{HH}}=1.7 \mathrm{~Hz},{ }^{3} J_{\mathrm{HH}}=0.6 \mathrm{~Hz}, 3 \mathrm{H}, 3-H_{\mathrm{pz}}\right) \mathrm{ppm} .{ }^{13} \mathrm{C}\left\{{ }^{1} \mathrm{H}\right\}$ NMR $\left(75.48 \mathrm{MHz}, \mathrm{C}_{6} \mathrm{D}_{6}\right): \delta=19.3\left(\mathrm{~d},{ }^{2} J_{\mathrm{CP}}=13.9 \mathrm{~Hz}\right.$, $\left.\mathrm{CH}-\left(\mathrm{CH}_{3}\right)_{2}\right), 22.3\left(\mathrm{~d},{ }^{2} J_{\mathrm{CP}}=24.2 \mathrm{~Hz}, \mathrm{CH}-\left(\mathrm{CH}_{3}\right)_{2}\right), 24.2\left(\mathrm{~d},{ }^{1} J_{\mathrm{CP}}=\right.$ $\left.24.9 \mathrm{~Hz}, \mathrm{CH}-\left(\mathrm{CH}_{3}\right)_{2}\right), 95.2\left(\mathrm{~d},{ }^{1} J_{\mathrm{CP}}=55.8 \mathrm{~Hz}, C_{\text {apical }}\right), 106.3(\mathrm{~d}$, $\left.{ }^{4} J_{\mathrm{CP}}=1.5 \mathrm{~Hz}, 4-C_{\mathrm{pz}}\right), 130.3\left(\mathrm{~d},{ }^{3} J_{\mathrm{CP}}=1.5 \mathrm{~Hz}, 5-C_{\mathrm{pz}}\right), 140.6(\mathrm{~s}$, 3- $\left.C_{\mathrm{pz}}\right)$ ppm. ${ }^{31} \mathrm{P}\left\{{ }^{1} \mathrm{H}\right\}$ NMR (121.49 $\left.\mathrm{MHz}, \mathrm{C}_{6} \mathrm{D}_{6}\right): \delta=57.9$ (s) ppm. EA [\%] calc. $\mathrm{C}_{16} \mathrm{H}_{27} \mathrm{~N}_{6} \mathrm{P}$ : C 58.18, $\mathrm{H}$ 7.02, N 25.44; found C $57.63, \mathrm{H}$ 6.65, N 25.54. APCI-MS $(m / z)$ : calc. 330.17 , found $331.4[\mathrm{M}+\mathrm{H}]^{+}$. FT-IR (solid, ATR, $\mathrm{cm}^{-1}$ ): 3117 (vw), $3001(\mathrm{vw})$, $2958(\mathrm{w}), 2940(\mathrm{vw}), 2868(\mathrm{w}), 1582(\mathrm{w}), 1511(\mathrm{~m}), 1471(\mathrm{w})$, 1448 (m), 1415 (s), 1385 (m), 1379 (s), 1312 (m), 1295 (m), $1235(\mathrm{~m}), 1189(\mathrm{~m}), 1089(\mathrm{~s}), 1082(\mathrm{~s}), 1041(\mathrm{~s}), 970(\mathrm{~m})$, 914 (w), 888 (s), 851 (w), 834 (vs), 758 (vs), 745 (vs), 649 (m), $618(\mathrm{~m}), 609$ (m), $594(\mathrm{~m}), 484(\mathrm{~m})$. Mp (sealed tube under Ar): $124-126^{\circ} \mathrm{C}$.

Synthesis of $3 \mathrm{a} . \mathrm{TpmPPh}_{2}(102.4 \mathrm{mg} ; 0.257 \mathrm{mmol})$ and $\left[\mathrm{Rh}(\mathrm{CO})_{2} \mathrm{Cl}\right]_{2}(50.0 \mathrm{mg} ; 0.129 \mathrm{mmol})$ are dissolved in dichloromethane $(10 \mathrm{~mL})$ and a yellow solution is obtained. The solution is stirred overnight. After adding $10 \mathrm{~mL}$ of hexane, yellow crystals are obtained. Yield: $117 \mathrm{mg}(81 \%) .{ }^{1} \mathrm{H}$ NMR $(300 \mathrm{MHz}$, $\left.\mathrm{CD}_{2} \mathrm{Cl}_{2}\right): \delta=6.25\left(\mathrm{t},{ }^{3} \mathrm{~J}_{\mathrm{HH}}=2.3 \mathrm{~Hz}, 3 \mathrm{H}, 4-H_{\mathrm{pz}}\right), 7.20-7.34(\mathrm{~m}$, $\left.4 \mathrm{H}, H_{\mathrm{Ph}}\right), 7.35-7.47\left(\mathrm{~m}, 2 \mathrm{H}, H_{\mathrm{Ph}}\right), 7.50\left(\mathrm{dd},{ }^{3} J_{\mathrm{HH}}=2.8 \mathrm{~Hz}\right.$, $\left.{ }^{3} J_{\mathrm{HH}}=0.7 \mathrm{~Hz}, 3 \mathrm{H}, 3-H_{\mathrm{pz}}\right), 7.73\left(\mathrm{br}, 3 \mathrm{H}, 5-H_{\mathrm{pz}}\right), 7.83-8.00(\mathrm{~m}$, $\left.4 \mathrm{H}, H_{P h}\right)$ ppm. ${ }^{13} \mathrm{C}\left\{{ }^{1} \mathrm{H}\right\}$ NMR $\left(75.48 \mathrm{MHz}, \mathrm{CD}_{2} \mathrm{Cl}_{2}\right): \delta=94.80(\mathrm{~d}$, $\left.{ }^{1} J_{\mathrm{CP}}=24.7 \mathrm{~Hz}, C_{\text {apical }}\right), 98.26\left(\mathrm{~d},{ }^{1} J_{\mathrm{CRh}}=1.7 \mathrm{~Hz}, \mathrm{C}_{\mathrm{Ph}}\right), 108.53(\mathrm{~s}$, $\left.C_{\mathrm{pz}}\right), 128.46\left(\mathrm{~d},{ }^{2} J_{\mathrm{CRh}}=11.5 \mathrm{~Hz}, C_{\mathrm{pz}}\right), 128.81\left(\mathrm{dd},{ }^{1} J_{\mathrm{CP}}=50.9\right.$, $\left.{ }^{2} J_{\mathrm{CRh}}=1.9 \mathrm{~Hz}, C_{\mathrm{Ph}}\right), 132.07-132.29\left(\mathrm{~m}, C_{\mathrm{Ph}}\right), 135.55\left(\mathrm{dd},{ }^{2} J_{\mathrm{CP}}=\right.$ $\left.14.1,{ }^{3} J_{\mathrm{CRh}}=1.4 \mathrm{~Hz}, C_{\mathrm{Ph}}\right), 142.92\left(\mathrm{~s}, C_{\mathrm{pz}}\right), 189.54\left(\mathrm{dd},{ }^{1} J_{\mathrm{CRh}}=\right.$ 72.2, $\left.{ }^{2} J_{\mathrm{CP}} 14.9 \mathrm{~Hz}, \mathrm{CO}\right) \mathrm{ppm} .{ }^{31} \mathrm{P}\left\{{ }^{1} \mathrm{H}\right\} \mathrm{NMR}\left(121 \mathrm{MHz}, \mathrm{CD}_{2} \mathrm{Cl}_{2}\right)$ : $\delta=112.83\left(\mathrm{~d},{ }^{1} J_{\mathrm{PRh}}=170.3 \mathrm{~Hz}\right)$ ppm. EA [\%] calc. $\mathrm{C}_{23} \mathrm{H}_{19} \mathrm{ClN}_{6} \mathrm{OPRh}$ : C 48.91, H 3.39, N 14.88; found: C 49.50, $\mathrm{H} 3.33, \mathrm{~N}$ 14.65. APCI-MS $(\mathrm{m} / \mathrm{z})$ : calc. 564.01 , found 565.2 $[\mathrm{M}+\mathrm{H}]^{+}$. FT-IR (solid, ATR, $\mathrm{cm}^{-1}$ ): 3143 (vw), 3125 (vw), $3050(\mathrm{vw}), 2212(\mathrm{vw}), 2176(\mathrm{vw}), 2130(\mathrm{vw}), 2056(\mathrm{vw}), 2030(\mathrm{vw})$, 1994 (vs), 1952 (vw), 1517 (vw), 1475 (vw), 1431 (w), 1414 (vw), $1395(\mathrm{vw}), 1379(\mathrm{w}), 1321(\mathrm{w}), 1307(\mathrm{w}), 1247(\mathrm{w}), 1195(\mathrm{w})$, 1180 (vw), 1155 (vw), 1082 (m), 1069 (w), 1039 (w), 987 (vw), $970(\mathrm{vw}), 957(\mathrm{vw}), 912(\mathrm{vw}), 885(\mathrm{w}), 850(\mathrm{w}), 839(\mathrm{~m}), 773(\mathrm{~m})$, $750(\mathrm{vs}), 694(\mathrm{vs}), 665(\mathrm{vw}), 639(\mathrm{w}), 620(\mathrm{vw}), 602(\mathrm{vw}), 586(\mathrm{~s})$, 520 (m), 507 (vs), 496 (vs), 479 (vs), 440 (w), 417 (s), 408 (m), 386 (vw). Mp (sealed tube under Ar): $244{ }^{\circ} \mathrm{C}$. 
Synthesis of $3 \mathbf{b}$. $\mathrm{TpmPBu}_{2}(100 \mathrm{mg} ; 0.279 \mathrm{mmol})$ and $\left[\mathrm{Rh}(\mathrm{CO})_{2} \mathrm{Cl}\right]_{2}(54.2 \mathrm{mg} ; 0.139 \mathrm{mmol})$ are dissolved in tetrahydrofurane $(5 \mathrm{~mL})$ and a yellow solution is obtained. After stirring over night at room temperature the solution turns orange. After adding $10 \mathrm{~mL}$ of hexane, yellow crystals are obtained. Yield: $128 \mathrm{mg}$ (87\%). ${ }^{1} \mathrm{H}$ NMR (400.13 MHz, thf- $\left.\mathrm{d}_{8}\right): \delta=0.85(\mathrm{t}$, $\left.{ }^{3} J_{\mathrm{HH}}=7.2 \mathrm{~Hz}, 6 \mathrm{H}, \mathrm{CH}_{3}\right), 1.29-1.36\left(\mathrm{~m}, 4 \mathrm{H}, \mathrm{CH}_{2}\right), 1.37-1.44(\mathrm{~m}$, 2H, $\left.\mathrm{CH}_{2}\right), 1.46-1.58\left(\mathrm{~m}, 2 \mathrm{H}, \mathrm{CH}_{2}\right), 1.92-2.13\left(\mathrm{~m}, 4 \mathrm{H}, \mathrm{CH}_{2}\right), 6.51$ $\left(\mathrm{dd},{ }^{3} J_{\mathrm{HH}}=2.8 \mathrm{~Hz},{ }^{3} J_{\mathrm{HH}}=1.9 \mathrm{~Hz}, 3 \mathrm{H}, 4-H_{\mathrm{pz}}\right), 7.52\left(\mathrm{~d},{ }^{3} J_{\mathrm{HH}}=\right.$ $\left.2.8 \mathrm{~Hz}, 3 \mathrm{H}, 3-H_{\mathrm{pz}}\right), 8.00\left(\mathrm{br}, 3 \mathrm{H}, 5-H_{\mathrm{pz}}\right) \mathrm{ppm} .{ }^{13} \mathrm{C}\left\{{ }^{1} \mathrm{H}\right\} \mathrm{NMR}$ (100.61 MHz, thf-d $\left.\mathrm{d}_{8}\right): \delta=13.96\left(\mathrm{~s}, \mathrm{CH}_{3}\right), 28.95,\left(\mathrm{~s}, \mathrm{CH}_{2}\right), 28.96$ $\left(\mathrm{d},{ }^{2} J_{\mathrm{CP}}=2.0 \mathrm{~Hz}, C \mathrm{H}_{2}\right), 29.19\left(\mathrm{~d},{ }^{1} J_{\mathrm{CP}}=2 \mathrm{~Hz}\right), 93.4\left(\mathrm{~d},{ }^{1} J_{\mathrm{CP}}=\right.$ $\left.20.93 \mathrm{~Hz}, C_{\text {apical }}\right), 108.91\left(\mathrm{~s}, C_{\mathrm{pz}}\right), 132.97\left(\mathrm{~s}, C_{\mathrm{pz}}\right), 143.16\left(\mathrm{~s}, C_{p z}\right)$, $191.62\left(\mathrm{dd},{ }^{1} J_{\mathrm{CRh}}=74.23 \mathrm{~Hz},{ }^{2} J_{\mathrm{CP}}=15.08 \mathrm{~Hz}, C \mathrm{O}\right) \mathrm{ppm} .{ }^{31} \mathrm{P}\left\{{ }^{1} \mathrm{H}\right\}$ NMR (121.49 MHz, thf-d $\left.{ }_{8}\right): \delta=114.04\left(\mathrm{~d}^{1} J_{\mathrm{PRh}}=155.4 \mathrm{~Hz}\right)$ ppm. EA [\%] calc. $\mathrm{C}_{19} \mathrm{H}_{27} \mathrm{ClN}_{6} \mathrm{OPRh}$ : C 43.49, H 5.19, N 16.01; found $\mathrm{C} 43.39, \mathrm{H}$ 4.93, 15.93. APCI-MS ( $\mathrm{m} / \mathrm{z})$ : calc. 524.07, found $525.3[\mathrm{M}+\mathrm{H}]^{+}$. FT-IR (solid, ATR, $\mathrm{cm}^{-1}$ ): $3114(\mathrm{vw}), 3094$ (vw), 2955 (vw), 2930 (vw), 2871 (vw), 2235 (vw), 2187 (vw), 2153 (vw), 2072(vw), 2048 (vw), 2019 (vw), 2008 (vw), 1981 (vs), 1517 (vw), 1457 (vw), 1430 (vw), 1419 (vw), 1377 (m), 1325 (w), 1312 (w), 1245 (w), 1226 (vw), 1204 (w), 1191 (vw), 1099 (w), 1086 (m), 1068 (w), 1041 (w), 990 (vw), 963 (vw), 935 (vw), 913(w), 889 (vw), $848(\mathrm{~m}), 838(\mathrm{w}), 788(\mathrm{~m}), 750$ (s), 661 (vw), 637 (vw), $622(\mathrm{w}), 605(\mathrm{w}), 581(\mathrm{~m}), 507(\mathrm{w}), 482(\mathrm{w}), 450(\mathrm{vw}), 406(\mathrm{vw})$, 381 (vw). Mp (sealed tube under Ar): 148.1-153.9 ${ }^{\circ} \mathrm{C}$.

Synthesis of 3c. $\operatorname{TpmPiPr}_{2}(165.2 \mathrm{mg} ; 0.5 \mathrm{mmol})$ and $\left[\mathrm{Rh}(\mathrm{CO})_{2} \mathrm{Cl}\right]_{2}$ (97.2 mg; $0.25 \mathrm{mmol}$ ) are dissolved in tetrahydrofurane $(10 \mathrm{~mL})$ and a yellow solution is obtained. The solution is stirred overnight. After adding $10 \mathrm{~mL}$ of hexane, yellow crystals are obtained. Yield: $212 \mathrm{mg}(85 \%) .{ }^{1} \mathrm{H}$ NMR $(300 \mathrm{MHz}$, thf$\left.\mathrm{d}_{8}\right): \delta=1.03\left(\mathrm{dd},{ }^{3} J_{\mathrm{HP}}=17.2 \mathrm{~Hz},{ }^{3} J_{\mathrm{HH}}=7.0 \mathrm{~Hz}, 6 \mathrm{H}, \mathrm{CH}-\left(\mathrm{CH}_{3}\right)_{2}\right)$, $1.17\left(\mathrm{dd},{ }^{3} J_{\mathrm{HP}}=17.4 \mathrm{~Hz},{ }^{3} J_{\mathrm{HH}}=7.1 \mathrm{~Hz}, 6 \mathrm{H}, \mathrm{CH}-\left(\mathrm{CH}_{3}\right)_{2}\right), 3.17$ $\left(\mathrm{ds},{ }^{2} J_{\mathrm{HP}}=10.9 \mathrm{~Hz},{ }^{3} J_{\mathrm{HH}}=7.1 \mathrm{~Hz}, 2 \mathrm{H}, \mathrm{CH}-\left(\mathrm{CH}_{3}\right)_{2}\right), 6.52(\mathrm{dd}$, $\left.{ }^{3} J_{\mathrm{HH}}=2.8 \mathrm{~Hz},{ }^{3} J_{\mathrm{HH}}=1.9 \mathrm{~Hz}, 3 \mathrm{H}, 4-H_{p z}\right), 7.23\left(\mathrm{dd},{ }^{3} J_{\mathrm{HH}}=2.8 \mathrm{~Hz}\right.$, $\left.{ }^{3} J_{\mathrm{HH}}=0.7 \mathrm{~Hz}, 3 \mathrm{H}, 3 H_{\mathrm{pz}}\right), 8.01\left(\mathrm{~d},{ }^{3} J_{\mathrm{HH}}=1.9 \mathrm{~Hz}, 3 \mathrm{H}, H_{\mathrm{pz}}\right) \mathrm{ppm}$. ${ }^{13} \mathrm{C}\left\{{ }^{1} \mathrm{H}\right\}$ NMR (100.61 MHz, thf-d $\left.{ }_{8}\right): \delta=19.88\left(\mathrm{~d},{ }^{3} J_{\mathrm{CP}}=3.4 \mathrm{~Hz}\right.$, $\left.\mathrm{CH}-\left(\mathrm{CH}_{3}\right)_{2}\right), 20.41$ (d, $\left.{ }^{3} J_{\mathrm{CP}}=1.9 \mathrm{~Hz}, \mathrm{CH}-\left(\mathrm{CH}_{3}\right)_{2}\right), 29.10$ (dd, $\left.{ }^{1} J_{\mathrm{CP}}=18.3,{ }^{2} J_{\mathrm{CRh}}=1.8 \mathrm{~Hz}, \mathrm{CH}-\left(\mathrm{CH}_{3}\right)_{2}\right), 94.24\left(\mathrm{~d},{ }^{1} J_{\mathrm{CP}}=11.8 \mathrm{~Hz}\right.$, $\left.C_{\text {apical }}\right), 109.08\left(\mathrm{~s}, C_{\mathrm{pz}}\right), 132.91\left(\mathrm{~s}, \mathrm{C}_{\mathrm{pz}}\right), 143.07\left(\mathrm{~s}, C_{\mathrm{pz}}\right), 192.21$ $\left(\mathrm{dd},{ }^{1} J_{\mathrm{CRh}}=73.5,{ }^{2} J_{\mathrm{CP}}=14.5 \mathrm{~Hz}, C \mathrm{O}\right) \mathrm{ppm} .{ }^{31} \mathrm{P}\left\{{ }^{1} \mathrm{H}\right\} \mathrm{NMR}$ $\left(121.49 \mathrm{MHz}\right.$, thf- $\left.\mathrm{d}_{8}\right): \delta=143.58\left(\mathrm{~d}^{1} J_{\mathrm{PRh}}=156.1 \mathrm{~Hz}\right) \mathrm{ppm}$. EA [\%] calc. $\mathrm{C}_{17} \mathrm{H}_{23} \mathrm{ClN}_{6} \mathrm{OPRh}$ : C 41.11, $\mathrm{H} 4.67, \mathrm{~N} 16.90$; found: $\mathrm{C}$ 41.13, H 4.58, N 16.90. APCI-MS $(\mathrm{m} / \mathrm{z})$ : calc. 496.04, found $497.3[\mathrm{M}+\mathrm{H}]^{+}$. FT-IR (solid, ATR, $\mathrm{cm}^{-1}$ ): 3158 (vw), 3122 (vw), 2958 (vw), 2937 (vw), 2230 (vw), 2174 (vw), 2125 (vw), 2020 (vw), 1978 (s), 1933 (vw), 1517 (vw), 1467 (vw), 1453 (vw), 1434 (vw), 1420 (vw), 1411 (vw), 1395 (w), 1378 (m), 1333 (w), 1319 (m), $1303(\mathrm{w}), 1234(\mathrm{~m}), 1201(\mathrm{w}), 1153(\mathrm{vw}), 1102(\mathrm{~m}), 1082(\mathrm{w})$, 1068 (m), 1043 (m), 1030 (w), 990 (vw), $969(\mathrm{w}), 913(\mathrm{w}), 882$ (w), 866 (m), 839 (s), 769 (vs), 745 (vs), 668 (w), 659 (m), 648 (w), 631 (s), 605 (s), 582 (m), 520 (w), 494 (s), 462 (m), 437 (vw), $405(\mathrm{w}), 384(\mathrm{w}) . \mathrm{Mp}$ (sealed tube under Ar): $216^{\circ} \mathrm{C}$.

Synthesis of 4. $\mathrm{TpmiPr}_{2}(37.7 \mathrm{mg} ; 0.115 \mathrm{mmol})$ and $[\mathrm{CpRu}$ $\left.(\mathrm{acn})_{3}\right]\left[\mathrm{PF}_{6}\right]$ (50 mg; $0.115 \mathrm{mmol}$ ) are dissolved in dichloromethane $(3 \mathrm{~mL})$ and an orange solution is obtained After adding $10 \mathrm{~mL}$ of hexane as antisolvent orange crystals are obtained. Yield: $67 \mathrm{mg}(91 \%) .{ }^{1} \mathrm{H}$ NMR $\left(300 \mathrm{MHz}, \mathrm{CD}_{2} \mathrm{Cl}_{2}\right): \delta=$ $0.57\left(\mathrm{dd},{ }^{3} J_{\mathrm{HP}}=16.6 \mathrm{~Hz},{ }^{3} J_{\mathrm{HH}}=7.1 \mathrm{~Hz}, 6 \mathrm{H}, \mathrm{CH}-\left(\mathrm{CH}_{3}\right)_{2}\right), 1.24$ $\left(\mathrm{dd},{ }^{3} J_{\mathrm{HP}}=17.5 \mathrm{~Hz},{ }^{3} J_{\mathrm{HH}}=7.0 \mathrm{~Hz}, 6 \mathrm{H}, \mathrm{CH}-\left(\mathrm{CH}_{3}\right)_{2}\right), 2.63$ (dhept, $\left.{ }^{2} J_{\mathrm{HP}}=11.7 \mathrm{~Hz},{ }^{3} J_{\mathrm{HH}}=7.1 \mathrm{~Hz}, 2 \mathrm{H}, \mathrm{CH}-\left(\mathrm{CH}_{3}\right)_{2}\right), 4.88(\mathrm{~s}, 5 \mathrm{H}$, $\left.H_{\mathrm{Cp}}\right), 6.46\left(\mathrm{dd},{ }^{3} J_{\mathrm{HH}}=3.0 \mathrm{~Hz},{ }^{3} J_{\mathrm{HH}}=2.2 \mathrm{~Hz}, 2 \mathrm{H}, H_{\mathrm{pz}}\right), 6.90(\mathrm{dd}$, $\left.{ }^{3} J_{\mathrm{HH}}=2.7 \mathrm{~Hz},{ }^{3} J_{\mathrm{HH}}=1.8 \mathrm{~Hz}, 1 \mathrm{H}, H_{\mathrm{pz}}\right), 7.57\left(\mathrm{dd},{ }^{3} J_{\mathrm{HH}}=3.0 \mathrm{~Hz}\right.$, $\left.{ }^{3} J_{\mathrm{HH}}=0.7 \mathrm{~Hz}, 2 \mathrm{H}, H_{\mathrm{pz}}\right), 7.96\left(\mathrm{dd},{ }^{3} J_{\mathrm{HH}}=1.9 \mathrm{~Hz},{ }^{3} J_{\mathrm{HH}}=0.5 \mathrm{~Hz}\right.$, $\left.1 \mathrm{H}, H_{\mathrm{pz}}\right), 8.04\left(\mathrm{dt},{ }^{3} J_{\mathrm{HH}}=2.2 \mathrm{~Hz},{ }^{3} \mathrm{~J}_{\mathrm{HH}}=0.5 \mathrm{~Hz}, 2 \mathrm{H}, H_{\mathrm{pz}}\right), 8.28$ $\left(\mathrm{dd},{ }^{3} J_{\mathrm{HH}}=2.8 \mathrm{~Hz},{ }^{3} J_{\mathrm{HH}}=0.5 \mathrm{~Hz}, 1 \mathrm{H}, H_{\mathrm{pz}}\right) .{ }^{13} \mathrm{C}\left\{{ }^{1} \mathrm{H}\right\} \mathrm{NMR}$ (75.48 MHz, $\left.\mathrm{CD}_{2} \mathrm{Cl}_{2}\right): \delta=19.0\left(\mathrm{~d},{ }^{2} J_{\mathrm{CP}}=4.0 \mathrm{~Hz}, \mathrm{CH}-\left(\mathrm{CH}_{3}\right)_{2}\right)$, $20.2\left(\mathrm{~d},{ }^{2} J_{\mathrm{CP}}=5.7 \mathrm{~Hz}, \mathrm{CH}-\left(\mathrm{CH}_{3}\right)_{2}\right), 28.3\left(\mathrm{~d},{ }^{1} J_{\mathrm{CP}}=12.3 \mathrm{~Hz}, \mathrm{CH}-\right.$ $\left.\left(\mathrm{CH}_{3}\right)_{2}\right), 74.9\left(\mathrm{~d},{ }^{2} J_{\mathrm{CP}}=2.4 \mathrm{~Hz}, C_{\mathrm{Cp}}\right), 95.2\left(\mathrm{~d},{ }^{1} J_{\mathrm{CP}}=5.3 \mathrm{~Hz}\right.$, $\left.C_{\text {apical }}\right), 110.3\left(\mathrm{~s}, C_{\mathrm{pz}}\right), 111.8\left(\mathrm{~s}, C_{\mathrm{pz}}\right), 129.6\left(\mathrm{~s}, C_{\mathrm{pz}}\right), 132.5(\mathrm{~s}$, $\left.C_{\mathrm{pz}}\right), 143.4\left(\mathrm{~s}, C_{\mathrm{pz}}\right), 148.6\left(\mathrm{~s}, C_{\mathrm{pz}}\right) \mathrm{ppm} .{ }^{31} \mathrm{P}\left\{{ }^{1} \mathrm{H}\right\} \mathrm{NMR}(121 \mathrm{MHz}$, $\mathrm{CD}_{2} \mathrm{Cl}_{2}$ ): $\delta=-144.4$ (sept, ${ }^{1} J_{\mathrm{PF}}=710.5 \mathrm{~Hz}, \mathrm{PF}_{6}{ }^{-}$), $170.4(\mathrm{~s}$, TpmP) ppm. ${ }^{19} \mathrm{~F}$ NMR (282.38 MHz, $\left.\mathrm{CD}_{2} \mathrm{Cl}_{2}\right) \delta=-73.20$ (d, $\left.{ }^{1} J_{\mathrm{FP}}=710.4 \mathrm{~Hz}, \mathrm{PF}_{6}{ }^{-}\right)$ppm. EA [\%] calc. $\mathrm{C}_{21} \mathrm{H}_{28} \mathrm{~F}_{6} \mathrm{~N}_{6} \mathrm{P}_{2} \mathrm{Ru}: \mathrm{C}$ 39.32, H 4.40, N 13.10; found: C 39.39, H 4.36, N 12.94. ESI-MS $(\mathrm{m} / \mathrm{z})$ : calc. $497.12\left[\mathrm{M}-\mathrm{PF}_{6}{ }^{-}\right]$found: 497.2. FT-IR (solid, ATR, $\left.\mathrm{cm}^{-1}\right): \nu=388(\mathrm{vw}), 411(\mathrm{vw}), 440(\mathrm{vw}), 483(\mathrm{~m}), 555(\mathrm{vs}), 590$ (vw), $604(\mathrm{vw}), 655(\mathrm{w}), 668(\mathrm{w}), 738(\mathrm{w}), 763(\mathrm{w}), 832$ (vs), 875 (vw), 923 (vw), 955 (vw), 985 (vw), 1029 (vw), 1052 (w), $1091(\mathrm{w})$, 1108 (vw), 1158 (vw), 1194 (vw), 1211 (vw), 1220 (vw), 1256 (vw), 1320 (w), 1339 (w), 1383 (w), 1406 (vw), 1428 (vw), 1465 (vw), 1529 (vw), 1959 (vw), 1982 (vw), 2022 (vw), 2168 (vw), 2875 (vw), 2968 (vw), 3149 (vw). Mp (sealed tube under Ar): $235^{\circ} \mathrm{C}$ (dec.).

Synthesis of 5 . $\mathrm{TpmPPh}_{2}(300 \mathrm{mg}, 0.753 \mathrm{mmol})$ and $[($ tht $)$ AuCl] (241.4 mg, $0.753 \mathrm{mmol})$ are dissolved in THF $(10 \mathrm{~mL})$ and stirred overnight. The solvent is reduced to about $3 \mathrm{~mL}$ THF and hexane is added. The product is obtained as colourless crystals. Yield: $389 \mathrm{mg}$ (82\%). ${ }^{1} \mathrm{H}$ NMR (300.13 MHz, THF$\left.\mathrm{d}_{8}\right): \delta=6.29\left(\mathrm{t},{ }^{3} J_{\mathrm{HH}}=2.2 \mathrm{~Hz}, 3 \mathrm{H}, 4-H_{\mathrm{pz}}\right), 6.80\left(\mathrm{~d},{ }^{3} J_{\mathrm{HH}}=2.7 \mathrm{~Hz}\right.$, $\left.3 \mathrm{H}, 5-H_{\mathrm{pz}}\right), 7.27\left(\mathrm{~m}, 4 \mathrm{H}, m-H_{\mathrm{Ar}}\right), 7.37\left(\mathrm{~m}, 2 \mathrm{H}, p-H_{\mathrm{Ar}}\right), 7.64(\mathrm{~d}$, $\left.{ }^{3} J_{\mathrm{HH}}=1.8 \mathrm{~Hz}, 3 \mathrm{H}, 3-H_{\mathrm{pz}}\right), 8.04\left(\mathrm{~m}, 4 \mathrm{H}, o-H_{\mathrm{Ar}}\right) \mathrm{ppm} .{ }^{13} \mathrm{C}\left\{{ }^{1} \mathrm{H}\right\}$ NMR (75.48 MHz, THF-d ${ }_{8}$ ): $\delta=93.92\left(\mathrm{~d},{ }^{1} J_{\mathrm{CP}}=50.7 \mathrm{~Hz}, 1 \mathrm{C}\right.$, $\left.\mathrm{C}_{\text {apical }}\right) 108.17\left(\mathrm{~s}, 3 \mathrm{C}, 4-C_{\mathrm{pz}}\right), 121.15\left(\mathrm{~s}, 3 \mathrm{C}, 5-\mathrm{C}_{\mathrm{pz}}\right), 128.83(\mathrm{~d}$, $\left.{ }^{3} J_{\mathrm{CP}}=11.02 \mathrm{~Hz}, 4 \mathrm{C}, m-C_{\mathrm{Ar}}\right), 129.55\left(\mathrm{~d},{ }^{1} J_{\mathrm{CP}}=54.3 \mathrm{~Hz} 1 \mathrm{C}, C_{\mathrm{ipso}}\right)$, $132.08\left(\mathrm{~s}, 3 \mathrm{C}, 3-C_{\mathrm{pz}}\right), 132.25\left(\mathrm{~d},{ }^{4} J_{\mathrm{CP}}=2.5 \mathrm{~Hz}, 2 \mathrm{C}, p-C_{\mathrm{Ph}}\right), 136.97$ $\left(\mathrm{d},{ }^{2} J_{\mathrm{CP}}=15.3 \mathrm{~Hz}, 4 \mathrm{C}, o-C_{\mathrm{Ph}}\right), 143.07$ (s, 3C, 3- $\left.C_{\mathrm{pz}}\right) \mathrm{ppm} .{ }^{31} \mathrm{P}\left\{{ }^{1} \mathrm{H}\right\}$ NMR (121.49 MHz, THF-d $_{8}$ ): $\delta=78.60$ (s) ppm. MS (EI) $\mathrm{m} / z$ (\%): 398.1 (0.74) $\left[\mathbf{2 a}^{+}\right], 219.9$ (24.43) $\left[\mathrm{Tpm}^{+}\right]$. FT-IR (solid, ATR, $\mathrm{cm}^{-1}$ ): $\nu=379$ (vw), 385 (w), 406 (vs), 415 (vs), 437 (s), 446 (vs), 463 (s), 481 (s), 506 (vs), 525 (vs), 534 (m), 547 (w), 562 (w), 572 (m), $584(\mathrm{~m}), 600(\mathrm{~m}), 622(\mathrm{w}), 653(\mathrm{w}), 686(\mathrm{w}), 709(\mathrm{w}), 744$ (m), $761(\mathrm{w}), 853(\mathrm{w}), 880(\mathrm{w}), 977$ (vw), 1047 (vw), 1083 (vw), 1096 (vw), 1185 (vw), 1245 (vw), 1322 (vw), 1384 (vw), 1435 (vw), 1479 (vw), 1515 (vw), 1933 (vw), 1965 (vw), 2001 (vw), 2028 (vw), 2071 (vw), 2087 (vw), 2123 (vw), 2177 (vw). EA [\%] calc. $\mathrm{C}_{22} \mathrm{H}_{19} \mathrm{AuClN}_{6} \mathrm{P}: \mathrm{C}$ 41.89, H 3.04, N 13.32, found: $\mathrm{C} 41.60, \mathrm{H}$ 2.99, $\mathrm{N}$ 13.04. $\mathrm{Mp}$ (sealed tube under Ar): $145{ }^{\circ} \mathrm{C}$ (decomposition).

Synthesis of 6. $\mathrm{TpmPPh}_{2}(300 \mathrm{mg}, 0.753 \mathrm{mmol})$ and $[\mathrm{Pd}(\text { allyl }) \mathrm{Cl}]_{2}(137.8 \mathrm{mg}, 0.3765 \mathrm{mmol})$ are dissolved in THF $(10 \mathrm{~mL})$ and stirred overnight. The solvent is removed and the residue is washed with $5 \mathrm{ml}$ of $\mathrm{Et}_{2} \mathrm{O}$. The yellow powder is dis- 
solved in THF ( $3 \mathrm{~mL}$ ) and after adding $10 \mathrm{~mL}$ of hexane, yellow crystals are obtained. Yield: $207 \mathrm{mg}$ (47\%). ${ }^{1} \mathrm{H} \quad \mathrm{NMR}$ $\left(300.13 \mathrm{MHz}, \mathrm{THF}_{8}\right): \delta=5.88\left(\mathrm{~m}, \mathrm{H}, 2-\mathrm{H}_{\text {allyl }}\right), 6.21\left(\mathrm{dd},{ }^{3} \mathrm{~J}_{\mathrm{HH}}=\right.$ $\left.1.81 \mathrm{~Hz},{ }^{3} J_{\mathrm{HH}}=0.8 \mathrm{~Hz}, 3 \mathrm{H}, 4-\mathrm{H}_{\mathrm{pz}}\right), 6,78(\mathrm{~m}, 3 \mathrm{H}, 5-\mathrm{Hpz}), 7.20$ $\left(\mathrm{m}, 4 \mathrm{H}, m-H_{\mathrm{Ar}}\right), 7.34\left(\mathrm{~m}, 2 \mathrm{H}, p-H_{\mathrm{Ar}}\right), 7.53\left(\mathrm{~d},{ }^{3} J_{\mathrm{HH}}=2.67 \mathrm{~Hz}, 3 \mathrm{H}\right.$, 3- $\left.\mathrm{H}_{\mathrm{pz}}\right), 7.87\left(\mathrm{~m}, 4 \mathrm{H}, o-H_{\mathrm{Ar}}\right) \mathrm{ppm} .{ }^{31} \mathrm{P}\left\{{ }^{1} \mathrm{H}\right\}$ NMR (121.49 MHz, THF- $\left.\mathrm{d}_{8}\right): \delta=89.4(\mathrm{~s}) \mathrm{ppm}$. Due to the low solubility of the pure product, no evaluable ${ }^{13} \mathrm{C}$ spectra could be obtained. EA [\%] calc. $\mathrm{C}_{25} \mathrm{H}_{24} \mathrm{ClN}_{6} \mathrm{PPd}$ : C 51.56, $\mathrm{H} 4.33, \mathrm{~N} 14.43$, found: $\mathrm{C} 51.56$, $\mathrm{H}$ 4.03, N 14.18. MS (EI) $\mathrm{m} / z$ (\%): 577.22 (1.89) $\left[\mathrm{M}^{+}\right], 219.9$ (16.09) $\left[\mathrm{Tpm}^{+}\right]$. FT-IR (solid, ATR, $\left.\mathrm{cm}^{-1}\right): \nu=378(\mathrm{vs}), 387(\mathrm{~m})$, 397 (vs), 416 (vs), 432 (s), 444 (m), 453 (s), 464 (m), 481 (s), 502 (vs), $520(\mathrm{~m}), 536$ (s), $549(\mathrm{~m}), 558(\mathrm{~m}), 569(\mathrm{~m}), 591(\mathrm{~m}), 600$ (m), $611(\mathrm{~m}), 624(\mathrm{w}), 644(\mathrm{w}), 654(\mathrm{w}), 664(\mathrm{w}), 692(\mathrm{~m}), 703$ (m), $721(\mathrm{w}), 740(\mathrm{~s}), 794(\mathrm{w}), 837(\mathrm{w}), 847(\mathrm{w}), 874(\mathrm{w}) 913(\mathrm{vw})$, $982(\mathrm{w}), 1044(\mathrm{w}), 1085$ (w), 1096 (w), 1133 (vw), 1197 (vw), 1238 (w), 1315 (vw), 1965 (vw), 1975 (vw), 1983 (vw), 2005 (vw), 2015 (vw), 2033 (vw), 2157 (vw), 2166 (vw), 2175 (vw). Mp (sealed tube under Ar): $240{ }^{\circ} \mathrm{C}$ (decomposition).

Synthesis of compound 7. $\mathrm{TpmPPh}_{2}(70 \mathrm{mg}, 0.176 \mathrm{mmol})$ and $\left[\mathrm{Pd}(\operatorname{cod}) \mathrm{Cl}_{2}\right](50 \mathrm{mg}, 0.176 \mathrm{mmol})$ are dissolved in THF $(10 \mathrm{~mL})$ and stirred overnight. After all volatiles are removed the residue is solved in THF again and after adding $15 \mathrm{~mL}$ of hexane, yellow crystals are obtained. Yield: $88 \mathrm{mg}(87 \%) .{ }^{1} \mathrm{H}$ NMR (300.13 MHz, $\left.\mathrm{CD}_{2} \mathrm{Cl}_{2}\right): \delta=6.09,\left(\mathrm{~s}, 2 \mathrm{H}, \mathrm{H}_{\mathrm{pz}}\right), 6.76(\mathrm{~s}, 1 \mathrm{H}$, coord. $\mathrm{H}_{\mathrm{pz}}$ ), $7.25\left(\mathrm{~s}, 2 \mathrm{H}, \mathrm{H}_{\mathrm{pz}}\right), 7.33\left(\mathrm{~m}, 4 \mathrm{H}, \mathrm{H}_{\mathrm{Ph}}\right), 7.48(\mathrm{~m}, 2 \mathrm{H}$, $\left.\mathrm{H}_{\mathrm{pz}}\right) 7.52\left(\mathrm{~m}, 2 \mathrm{H}, \mathrm{H}_{\mathrm{Ph}}\right), 7.57\left(\mathrm{~s}, 1 \mathrm{H}\right.$, coord. $\left.\mathrm{H}_{\mathrm{pz}}\right), 8.00(\mathrm{~m}, 4 \mathrm{H}$, $\mathrm{H}_{\mathrm{Ph}}$ ), $8.90\left(\mathrm{~s}, 1 \mathrm{H}\right.$, coord. $\mathrm{H}_{\mathrm{pz}}$ ) ppm. ${ }^{13} \mathrm{C} \mathrm{NMR}(75.47 \mathrm{MHz}$, $\mathrm{CD}_{2} \mathrm{Cl}_{2}$ ): $\delta=144.79\left(\mathrm{~s}, 2 \mathrm{C}, \mathrm{Cpz}_{\text {uncoord }} 143.50\right.$ (s, 1C, $\mathrm{Cpz}_{\text {coord }}$ ), $135.86\left(\mathrm{~d}, 4 \mathrm{C}\right.$, meta $\left.-\mathrm{C}_{\mathrm{Ph}},{ }^{3} J_{\mathrm{CP}}=12.3 \mathrm{~Hz}\right), 133.39$ (d, 2C, para$\left.\mathrm{C}_{\mathrm{Ph}},{ }^{4} J_{\mathrm{CP}}=2.9 \mathrm{~Hz}\right), 131.35\left(\mathrm{~s}, 2 \mathrm{C}, \mathrm{Cpz}_{\text {uncoord }}\right), 128.73$ (s, 1C, $C \mathrm{pz}_{\text {coord }}$ ), 129.01 (d, ${ }^{2} J_{\mathrm{CP}}=18.8 \mathrm{~Hz}, 4 \mathrm{C}$, ortho- $\left.\mathrm{C}_{\mathrm{Ph}}\right), 123.34$ (d, ${ }^{1} J_{\mathrm{CP}}=55.4 \mathrm{~Hz}, 2 \mathrm{C}$, ipso- $\left.\mathrm{C}_{\mathrm{Ph}}\right), 112.26\left(\mathrm{~s}, 2 \mathrm{C}, \mathrm{Cpz}_{\text {uncord. }}\right), 108.07$ (s, 1C, $\mathrm{Cpz}_{\text {coord }}$ ), 94.44 (d, $\left.{ }^{1} J_{\mathrm{CP}}=5.3 \mathrm{~Hz}, C_{\text {apical }}\right) \mathrm{ppm} .{ }^{31} \mathrm{P}\left\{{ }^{1} \mathrm{H}\right\}$ NMR (121 MHz, $\left.\mathrm{CD}_{2} \mathrm{Cl}_{2}\right) \delta=90.72$ (s) ppm. EA [\%] calc. $\mathrm{C}_{22} \mathrm{H}_{19} \mathrm{Cl}_{2} \mathrm{~N}_{6} \mathrm{PPd}$ : C 45.90, H 3.33, N 14.60, found: $\mathrm{C} 46.13, \mathrm{H}$ 3.35, N 14.10. ESI-MS (m/z): calc. $647.04[\mathrm{M}+\mathrm{H}+\mathrm{THF}]^{+}$found: 648.3, 497.2. FT-IR (solid, ATR, $\mathrm{cm}^{-1}$ ): $\nu=398$ (vw), $418(\mathrm{~m})$, 447 (w), 479 (s), 501 (vs), $536(\mathrm{~s}), 593(\mathrm{w}), 600(\mathrm{w}), 612(\mathrm{vw}), 624$ (vw), 652 (vw), 691 (m), 704 (m), 723 (w), 741 (vs), 795 (m), 840 (w), 851 (w), 875 (w), 896 (vw), 914 (vw), 926 (vw), 958 (vw), 970 (vw), 993 (w), 1025 (vw), 1044 (m), 1076 (w), 1085 (w), 1096 (m), 1164 (vw), 1186 (vw), 1198 (w), 1206 (vw), 1249 (vw), 1321 (m), 1381 (w), 1395 (w), 1418 (vw), 1434 (w), 1441 (w), 1480 (vw), 1516 (vw), 1587 (vw), 1820 (vw), 2181 (vw), 3008 (vw), 3065 (vw), 3130 (vw), 3144 (vw). Mp (sealed tube under Ar): $218^{\circ} \mathrm{C}$ (dec.).

Synthesis of compound 8.7 (30 mg, $0.052 \mathrm{mmol})$ and $\mathrm{CuCl}_{2}$ (7 $\mathrm{mg}, 0.052 \mathrm{mmol}$ ) are dissolved in $\mathrm{CH}_{2} \mathrm{Cl}_{2}(5 \mathrm{~mL})$ and stirred overnight while a blueish green solid precipitates. After all volatiles are removed, the residue is solved in MeCN $(5 \mathrm{~mL})$ and after addition of $\mathrm{Et}_{2} \mathrm{O}$, green crystals are obtained. Yield: $21 \mathrm{mg}$ (57\%). Paramagnetic compound, no NMR data recorded. FT-IR (solid, ATR, $\mathrm{cm}^{-1}$ ): $\nu=383(\mathrm{w}), 392(\mathrm{w}), 400$ (w), 418 (vw), 437 (vw), 456 (vw), 479 (s), 501 (vs), 526 (m), 601 (m), $612(\mathrm{vw}), 626(\mathrm{vw}), 636(\mathrm{vw}), 684(\mathrm{~s}), 696(\mathrm{w}), 720(\mathrm{w}), 743$ (s), $765(\mathrm{~m}), 782(\mathrm{~m}), 849(\mathrm{w}), 858(\mathrm{w}), 868(\mathrm{vw}), 911(\mathrm{vw}), 987$ (w), 1062 (w), 1075 (vw), 1088 (w), 1099 (m), 1165 (vw), 1194 (vw), 1206 (w), 1249 (vw), 1269 (vw), 1321 (w), 1333 (vw), 1379 (w), 1393 (w), 1429 (w), 1480 (vw), 1522 (vw), 1585 (vw), 1952 (vw), 2001 (vw), 2023 (vw), 2182 (vw), 2248 (vw), 3099 (vw), 3113 (vw). Mp (sealed tube under Ar): $132{ }^{\circ} \mathrm{C}$ (dec.).

\section{X-Ray crystallographic studies}

A suitable crystal was covered in mineral oil (Aldrich) and mounted on a glass fiber or a mylar loop. The crystal was transferred directly to the cold stream of a STOE IPDS 2 diffractometer.

All structures were solved by using the program SHELXS $/ \mathrm{T}^{21}$ using Olex2. ${ }^{22}$ The remaining non-hydrogen atoms were located from successive difference Fourier map calculations. The refinements were carried out by using full-matrix leastsquares techniques on $F^{2}$ by using the program Olex2. In each case, the locations of the largest peaks in the final difference Fourier map calculations, as well as the magnitude of the residual electron densities, were of no chemical significance. Details on the data refinement are deposited in the ESI.

Crystal data 2a. $\mathrm{C}_{22} \mathrm{H}_{19} \mathrm{~N}_{6} \mathrm{P}, M_{\mathrm{r}}=398.40$, orthorhombic, Pna2 ${ }_{1}$ (no. 33), $a=17.416(4) \AA, b=8.4390(17) \AA, c=40.806(8) \AA$, $V=5997(2) \AA^{3}, T=200 \mathrm{~K}, Z=12, \mu\left(\mathrm{MoK}_{\alpha}\right)=0.159,80621$ reflections measured, 11783 unique $\left(R_{\text {int }}=0.0935\right)$ which were used in all calculations. The final $\mathrm{w} R_{2}$ was 0.1631 (all data) and $R_{1}$ was $0.0580(I>2(I))$.

Crystal data 2 b. $\mathrm{C}_{18} \mathrm{H}_{27} \mathrm{~N}_{6} \mathrm{P}, M_{\mathrm{r}}=358.42$, triclinic, $P \overline{1}$ (no. 2), $a=8.1293(16) \AA, b=8.6311(17) \AA, c=15.582(3) \AA, \alpha=77.76(3)$, $\beta=86.99(3)^{\circ}, \gamma=66.40(3), V=978.5(4) \AA^{3}, T=200 \mathrm{~K}, Z=2$, $\mu\left(\mathrm{MoK}_{\alpha}\right)=0.154,18987$ reflections measured, 5275 unique $\left(R_{\mathrm{int}}=0.0458\right)$ which were used in all calculations. The final $\mathrm{w} R_{2}$ was 0.1196 (all data) and $R_{1}$ was $0.0408(I>2(I))$.

Crystal data 2c. $\mathrm{C}_{16} \mathrm{H}_{23} \mathrm{~N}_{6} \mathrm{P}, M_{\mathrm{r}}=330.37$, monoclinic, $P 2_{1} / c$ (no. 14), $a=8.9830(18) \AA, b=11.438(2) \AA, c=16.812(3) \AA, \beta=$ 97.07(3) $)^{\circ} V=1714.3(6) \AA^{3}, T=200 \mathrm{~K}, Z=4, \mu\left(\mathrm{MoK}_{\alpha}\right)=0.169$, 32515 reflections measured, 4632 unique $\left(R_{\text {int }}=0.0911\right)$ which were used in all calculations. The final $w R_{2}$ was 0.1014 (all data) and $R_{1}$ was $0.0393(I>2(I))$.

Crystal data 3a. $\mathrm{C}_{23} \mathrm{H}_{20} \mathrm{ClN}_{6} \mathrm{OPRh}, M_{\mathrm{r}}=565.78$, monoclinic, $P 2_{1} / c$ (no. 14), $a=10.430(2) \AA, b=15.442(3) \AA, c=14.382(3) \AA$, $\beta=104.04(3)^{\circ}, V=2247.2(8) \AA^{3}, T=200 \mathrm{~K}, Z=4, \mu\left(\mathrm{MoK}_{\alpha}\right)=$ $0.980,15146$ reflections measured, 4393 unique $\left(R_{\text {int }}=0.0375\right)$ which were used in all calculations. The final $\mathrm{w} R_{2}$ was 0.0648 (all data) and $R_{1}$ was $0.0240(I>2(I))$.

Crystal data $3 b . \mathrm{C}_{19} \mathrm{H}_{27} \mathrm{ClN}_{6} \mathrm{OPRh}, M_{\mathrm{r}}=524.79$, monoclinic, $P 2_{1} / c$ (no. 14), $a=17.969(4) \AA, b=8.9300(18) \AA, c=15.408(3) \AA$, $\beta=110.01(3)^{\circ}, V=2323.1(9) \AA^{3}, T=200 \mathrm{~K}, Z=4, \mu\left(\mathrm{MoK}_{\alpha}\right)=$ $0.941,14865$ reflections measured, 4548 unique $\left(R_{\text {int }}=0.1361\right)$ which were used in all calculations. The final $\mathrm{w} R_{2}$ was 0.1632 (all data) and $R_{1}$ was $0.0565(I>2(I))$.

Crystal data 3c. $\mathrm{C}_{17} \mathrm{H}_{23} \mathrm{ClN}_{6} \mathrm{OPRh}, M_{\mathrm{r}}=496.74$, monoclinic, $P 2_{1}$ (no. 4), $a=8.6320(17) \AA, b=12.927(3) \AA, c=10.003(2) \AA$, $\beta=114.42(3)^{\circ}, V=1016.3(4) \AA^{3}, T=200 \mathrm{~K}, Z=4, \mu\left(\mathrm{MoK}_{\alpha}\right)=$ 1.070, 19391 reflections measured, 5438 unique $\left(R_{\text {int }}=0.0528\right)$ which were used in all calculations. The final $\mathrm{w} R_{2}$ was 0.0687 (all data) and $R_{1}$ was $0.0290(I>2(I))$. 
Crystal data 4. $\mathrm{C}_{21} \mathrm{H}_{28} \mathrm{~F}_{6} \mathrm{~N}_{6} \mathrm{P}_{2} \mathrm{Ru}, M_{\mathrm{r}}=641.50$, orthorhombic, Pbcn (no. 60), $a=17.629(4) \AA, b=18.438(4) \AA, c=15.309(3) \AA$, $V=4976.1(17) \AA^{3}, T=200 \mathrm{~K}, Z=8, \mu\left(\mathrm{MoK}_{\alpha}\right)=0.827,91924$ reflections measured, 6718 unique $\left(R_{\text {int }}=0.0415\right)$ which were used in all calculations. The final $\mathrm{w} R_{2}$ was 0.0821 (all data) and $R_{1}$ was $0.0302(I>2(I))$.

Crystal data 5. $\mathrm{C}_{22} \mathrm{H}_{19} \mathrm{AuClN}_{6} \mathrm{P}, M_{\mathrm{r}}=630.82$, monoclinic, $P 2_{1} / n$ (no. 14), $a=8.7640(18) \AA, b=15.604(3) \AA ⿻$, $c=16.091(3) \AA$, $\beta=90.76(3)^{\circ}, V=2200.3(8) \AA^{3}, T=200 \mathrm{~K}, Z=4, \mu\left(\mathrm{MoK}_{\alpha}\right)=$ 1.904, 12511 reflections measured, 4760 unique $\left(R_{\text {int }}=0.0548\right)$ which were used in all calculations. The final $\mathrm{w} R_{2}$ was 0.1086 (all data) and $R_{1}$ was $0.0327(I>2(I))$.

Crystal data 6. $\mathrm{C}_{25} \mathrm{H}_{24} \mathrm{ClN}_{6} \mathrm{PPd}, M_{\mathrm{r}}=581.32$, orthorhombic, $P 2{ }_{1} 2_{1} 2_{1}$ (no. 19), $a=9.0270(18) \AA, b=15.942(3) \AA, c=17.225(3)$ $\AA, V=2478.8(9) \AA^{3}, T=200 \mathrm{~K}, Z=4, \mu\left(\mathrm{MoK}_{\alpha}\right)=1.558,9518$ reflections measured, 5395 unique $\left(R_{\text {int }}=0.0552\right)$ which were used in all calculations. The final $\mathrm{w} R_{2}$ was 0.1489 (all data) and $R_{1}$ was $0.0503(I>2(I))$.

Crystal data 7. $\mathrm{C}_{22} \mathrm{H}_{19} \mathrm{Cl}_{2} \mathrm{~N}_{6} \mathrm{PPd}, M_{\mathrm{r}}=575.70$, monoclinic, $P 2_{1} / c$ (no. 14), $a=14.818(3) \AA, b=9.2710(19) \AA, c=16.998(3) \AA$, $\beta=100.95(3)^{\circ}, V=2292.7(8) \AA^{3}, T=200 \mathrm{~K}, Z=4, \mu\left(\mathrm{MoK}_{\alpha}\right)=$ 1.136, 15955 reflections measured, 6154 unique $\left(R_{\text {int }}=0.0289\right)$ which were used in all calculations. The final $\mathrm{w} R_{2}$ was 0.0817 (all data) and $R_{1}$ was $0.0296(I>2(I))$.

Crystal data 8. $\mathrm{C}_{22} \mathrm{H}_{19} \mathrm{Cl}_{4} \mathrm{CuN}_{6} \mathrm{PPd} \cdot 2 \mathrm{C}_{2} \mathrm{H}_{3} \mathrm{~N}, M_{\mathrm{r}}=792.25$,

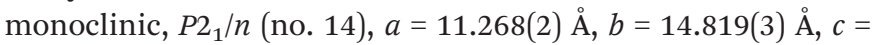
18.659(4) А, $\beta=98.36(3)^{\circ}, V=3082.6(11) \AA^{3}, T=200 \mathrm{~K}, Z=4$, $\mu\left(\mathrm{MoK}_{\alpha}\right)=1.704,18940$ reflections measured, 6055 unique $\left(R_{\text {int }}=0.0960\right)$ which were used in all calculations. The final $\mathrm{w} R_{2}$ was 0.1639 (all data) and $R_{1}$ was $0.0626(I>2(I)$ ).

Crystallographic data (excluding structure factors) for the structures reported in this paper have been deposited with the Cambridge Crystallographic Data Centre as a supplementary publication no. CCDC 1916045-1916055.

\section{Conflicts of interest}

There are no conflicts to declare.

\section{Acknowledgements}

We acknowledge financial support from the DFG-funded transregional collaborative research centre SFB/TRR 88 "Cooperative effects in homo- and heterometallic complexes (3MET)" (project B4). Support by the Federal Ministry of Education and Research (BMBF Project No. 02NUK020B) is also gratefully acknowledged.

\section{Notes and references}

1 (a) S. Trofimenko, Chem. Rev., 1993, 93, 943-980; (b) S. Trofimenko, Scorpionates: The Coordination Chemistry of Polypyrazolylborate Ligands, Imperial College Press, London, 1999.
2 (a) H. R. Bigmore, S. C. Lawrence, P. Mountford and C. S. Tredget, Dalton Trans., 2005, 635-651; (b) C. Pettinari and R. Pettinari, Coord. Chem. Rev., 2005, 249, 525-543.

3 (a) G. Parkin, in Adv. Inorg. Chem, ed. A. G. Sykes, Academic Press, 1995, vol. 42, pp. 291-393; (b) D. L. Reger, Coord. Chem. Rev., 1996, 147, 571-595; (c) M. Etienne, Coord. Chem. Rev., 1996, 156, 201-236; (d) C. Janiak, Coord. Chem. Rev., 1997, 163, 107-216; (e) D. L. Reger, Comments Inorg. Chem., 1999, 21, 1-28; $(f)$ N. Kitajima and W. B. Tolman, in Progress in Inorganic Chemistry, ed. K. D. Karlin, John Wiley \& Sons, Inc., 1995, vol. 43, p. 419; $(g)$ H. V. R. Dias and C. J. Lovely, Chem. Rev., 2008, 108, 3223-3238; (h) Adv. Organomet. Chem, ed. R. West, A. F. Hill and M. J. Fink, Academic Press, 2008, vol. 56, pp. 1-347; (i) S. Trofimenko, J. Chem. Educ., 2005, 82, 1715; (j) A. Munetaka and H. Shiro, Bull. Chem. Soc. Jpn., 2002, 75, 1657-1679; (k) G. Parkin, Chem. Rev., 2004, 104, 699-768.

4 (a) P. K. Byers, N. Carr and F. G. A. Stone, J. Chem. Soc., Dalton Trans., 1990, 3701-3708; (b) P. K. Byers and F. G. A. Stone, J. Chem. Soc., Dalton Trans., 1991, 93-99; (c) S. C. Lawrence, M. E. G. Skinner, J. C. Green and P. Mountford, Chem. Commun., 2001, 705-706; (d) F. Breher, J. Grunenberg, S. C. Lawrence, P. Mountford and H. Rüegger, Angew. Chem., Int. Ed., 2004, 43, 25212524; (e) H. R. Bigmore, S. R. Dubberley, M. Kranenburg, S. C. Lawrence, A. J. Sealey, J. D. Selby, M. A. Zuideveld, A. R. Cowley and P. Mountford, Chem. Commun., 2006, 436438; $(f)$ I. Krummenacher, H. Rüegger and F. Breher, Dalton Trans., 2006, 1073-1081; (g) H. R. Bigmore, J. Meyer, I. Krummenacher, H. Rüegger, E. Clot, P. Mountford and F. Breher, Chem. - Eur. J., 2008, 14, 5918-5934; (h) I. Kuzu, I. Krummenacher, I. J. Hewitt, Y. Lan, V. Mereacre, A. K. Powell, P. Höfer, J. Harmer and F. Breher, Chem. - Eur. J., 2009, 15, 4350-4365; (i) I. Fernández, P. Oña-Burgos, F. Armbruster, I. Krummenacher and F. Breher, Chem. Commun., 2009, 2586-2588; (j) M. G. Cushion, J. Meyer, A. Heath, A. D. Schwarz, I. Fernández, F. Breher and P. Mountford, Organometallics, 2010, 29, 1174-1190; (k) D. Kratzert, D. Leusser, D. Stern, J. Meyer, F. Breher and D. Stalke, Chem. Commun., 2011, 47, 2931-2933; (l) J. Meyer, I. Kuzu, S. González-Gallardo and F. Breher, Z. Anorg. Allg. Chem., 2013, 639, 301-307; (m) C. Müller, H. Görls, S. Krieck and M. Westerhausen, Eur. J. Inorg. Chem., 2013, 5679-5682; (n) S. González-Gallardo, I. Kuzu, P. OñaBurgos, T. Wolfer, C. Wang, K. W. Klinkhammer, W. Klopper, S. Bräse and F. Breher, Organometallics, 2014, 33, 941-951; (o) C. Müller, A. Koch, H. Görls, S. Krieck and M. Westerhausen, Inorg. Chem., 2015, 54, 635-645; ( $p$ ) C. Müller, S. Krieck, H. Görls and M. Westerhausen, Inorg. Chem., 2015, 54, 2100-2102; (q) R. Lalrempuia, A. Stasch and C. Jones, Chem. - Asian J., 2015, 10, 447454; (r) S. Krieck, A. Koch, K. Hinze, C. Müller, J. Lange, H. Görls and M. Westerhausen, Eur. J. Inorg. Chem., 2016, 2332-2348; $(s)$ I. Kuzu, A. Baldes, F. Weigend and F. Breher, Polyhedron, 2017, 125, 74-79. 
5 (a) F. Armbruster, I. Fernandez and F. Breher, Dalton Trans., 2009, 5612-5626; (b) F. Armbruster, T. Augenstein, P. Oña-Burgos and F. Breher, Chem. - Eur. J., 2013, 19, 17899-17906; (c) S. Styra, S. González-Gallardo, F. Armbruster, P. Oña-Burgos, E. Moos, M. Vonderach, P. Weis, O. Hampe, A. Grün, Y. Schmitt, M. Gerhards, F. Menges, M. Gaffga, G. Niedner-Schatteburg and F. Breher, Chem. - Eur. J., 2013, 19, 8436-8446; (d) M. Simon and F. Breher, Dalton Trans., 2017, 46, 79767997; (e) C. Krempner, M. H. Chisholm and J. Gallucci, Angew. Chem., Int. Ed., 2008, 47, 410-413; $(f)$ H. Li, L. J. Hope-Weeks and C. Krempner, Chem. Commun., 2011, 47, 4117-4119; (g) B. Carlson, A. J. A. Aquino, L. J. HopeWeeks, B. Whittlesey, B. McNerney, W. L. Hase and C. Krempner, Chem. Commun., 2011, 47, 11089-11091; (h) B. McNerney, B. Whittlesey and C. Krempner, Eur. J. Inorg. Chem., 2011, 1699-1702; (i) H. Li, F. Hung-Low and C. Krempner, Organometallics, 2012, 31, 7117-7124; (j) H. Li, A. J. A. Aquino, D. B. Cordes, W. L. Hase and C. Krempner, Chem. Sci., 2017, 8, 1316-1328.

6 (a) A. Steiner and D. Stalke, J. Chem. Soc., Chem. Commun., 1993, 1702-1704; (b) A. Steiner and D. Stalke, Inorg. Chem., 1995, 34, 4846-4853; (c) I. Krummenacher, C. Oschwald, H. Rüegger and F. Breher, Z. Anorg. Allg. Chem., 2007, 633, 2354-2361; (d) I. Krummenacher, I. Fernández, H. Rüegger, F. Weigend and F. Breher, Dalton Trans., 2009, 5335-5347; (e) M.-Á. MuñozHernández and V. Montiel-Palma, Inorg. Chim. Acta, 2009, 362, 4328-4339; $(f)$ H. R. Simmonds and D. S. Wright, Chem. Commun., 2012, 48, 8617-8624; $(g)$ K. Zeckert, Organometallics, 2013, 32, 1387-1393; (h) K. Zeckert, S. Zahn and B. Kirchner, Chem. Commun., 2010, 46, 26382640; (i) M. A. Halcrow, Dalton Trans., 2009, 2059-2073; (j) J. Klingele, S. Dechert and F. Meyer, Coord. Chem. Rev., 2009, 253, 2698-2741.

7 M. D. Ward, J. A. McCleverty and J. C. Jeffery, Coord. Chem. Rev., 2001, 222, 251-272.

8 R. F. Semeniuc and D. L. Reger, Eur. J. Inorg. Chem., 2016, 2253-2271.

9 (a) W. Kläui, M. Berghahn, G. Rheinwald and H. Lang, Angew. Chem., Int. Ed., 2000, 39, 2464-2466; (b) D. L. Reger, T. C. Grattan, K. J. Brown, C. A. Little, J. J. S. Lamba, A. L. Rheingold and R. D. Sommer, J. Organomet. Chem., 2000, 607, 120-128; (c) R. Wanke, P. Smoleński, M. F. C. Guedes da Silva, L. M. D. R. S. Martins and A. J. L. Pombeiro, Inorg. Chem., 2008, 47, 10158-10168; (d) D. L. Reger and T. C. Grattan, Synthesis, 2003, 03500356; (e) M. Müller, E. Lork and R. Mews, Angew. Chem., Int. Ed., 2001, 40, 1247-1249; (f) I. Trapp, S. GonzálezGallardo, S. Hohnstein, D. Garnier, P. Oña-Burgos and F. Breher, Dalton Trans., 2014, 43, 4313-4319; (g) T. Augenstein, F. Dorner, K. Reiter, H. E. Wagner, D. Garnier, W. Klopper and F. Breher, Chem. - Eur. J., 2016, 22, 7935-7943.

10 (a) I. Kuzu, I. Krummenacher, J. Meyer, F. Armbruster and F. Breher, Dalton Trans., 2008, 5836-5865; (b) F.-G. Fontaine, J. Boudreau and M.-H. Thibault, Eur. J. Inorg. Chem., 2008, 5439-5454.

11 (a) S. Maggini, Coord. Chem. Rev., 2009, 253, 1793-1832; (b) W.-H. Zhang, S. W. Chien and T. S. A. Hor, Coord. Chem. Rev., 2011, 255, 1991-2024.

12 (a) A. Otero, J. Fernández-Baeza, J. Tejeda, A. Antiñolo, F. Carrillo-Hermosilla, E. Díez-Barra, A. Lara-Sánchez, M. Fernández-López, M. Lanfranchi and M. A. Pellinghelli, J. Chem. Soc., Dalton Trans., 1999, 3537-3539; (b) A. Otero, J. Fernández-Baeza, A. Antiñolo, F. Carrillo-Hermosilla, J. Tejeda, A. Lara-Sánchez, L. Sánchez-Barba, M. Fernández-López, A. M. Rodríguez and I. López-Solera, Inorg. Chem., 2002, 41, 5193-5202; (c) A. Otero, J. Fernández-Baeza, A. Antiñolo, J. Tejeda, A. Lara-Sánchez, L. Sánchez-Barba, E. Martínez-Caballero, A. M. Rodríguez and I. López-Solera, Inorg. Chem., 2005, 44, 5336-5344; (d) A. Otero, J. Fernández-Baeza, A. Antiñolo, J. Tejeda, A. Lara-Sánchez, L. Sánchez-Barba, M. Fernández-López and I. López-Solera, Inorg. Chem., 2004, 43, 1350-1358; (e) A. Otero, J. Fernández-Baeza, A. Antiñolo, J. Tejeda, A. Lara-Sánchez, L. Sánchez-Barba and A. M. Rodríguez, Eur. J. Inorg. Chem., 2004, 260-266; $(f)$ A. Otero, J. Fernández-Baeza, A. Antiñolo, J. Tejeda and A. LaraSánchez, Dalton Trans., 2004, 1499-1510; (g) A. Otero, J. Fernández-Baeza, A. Lara-Sánchez, J. Tejeda and L. F. Sánchez-Barba, Eur. J. Inorg. Chem., 2008, 53095326.

13 (a) A. Beck, B. Weibert and N. Burzlaff, Eur. J. Inorg. Chem., 2001, 521-527; (b) S. Tampier, R. Müller, A. Thorn, E. Hübner and N. Burzlaff, Inorg. Chem., 2008, 47, 96249641; (c) H. Kopf, B. Holzberger, C. Pietraszuk, E. Hübner and N. Burzlaff, Organometallics, 2008, 27, 5894-5905; (d) H. Kopf, C. Pietraszuk, E. Hübner and N. Burzlaff, Organometallics, 2006, 25, 2533-2546; (e) R. Müller, E. Hübner and N. Burzlaff, Eur. J. Inorg. Chem., 2004, 21512159; $(f)$ N. V. Fischer, F. W. Heinemann and N. Burzlaff, Eur. J. Inorg. Chem., 2009, 3960-3965; (g) B. S. Hammes, M. T. Kieber-Emmons, J. A. Letizia, Z. Shirin, C. J. Carrano, L. N. Zakharov and A. L. Rheingold, Inorg. Chim. Acta, 2003, 346, 227-238.

14 S. Hohnstein, Vergleichende Studien zum Koordinationsverhalten tripodaler Liganden gegenüber f-Elementen sowie Synthese und Reaktivitätsstudien von neuartigen P,N-Hybridliganden, Dissertation, Logos Verlag Berlin $\mathrm{GmbH}$, Berlin, 2016.

15 (a) W. Kläui, M. Berghahn, W. Frank, G. J. Reiß, T. Schönherr, G. Rheinwald and H. Lang, Eur. J. Inorg. Chem., 2003, 2003, 2059-2070; (b) C. Pettinari, F. Marchetti, G. Lupidi, L. Quassinti, M. Bramucci, D. Petrelli, L. A. Vitali, M. F. C. Guedes da Silva, L. M. D. R. S. Martins, P. Smoleński and A. J. L. Pombeiro, Inorg. Chem., 2011, 50, 11173-11183; (c) F. Marchetti, C. Pettinari, R. Pettinari, A. Cerquetella, L. M. D. R. S. Martins, M. F. C. Guedes da Silva, T. F. S. Silva and A. J. L. Pombeiro, Organometallics, 2011, 30, 6180-6188.

16 C. A. Tolman, Chem. Rev., 1977, 77, 313-348. 
17 I. Kuzu, D. Nied and F. Breher, Eur. J. Inorg. Chem., 2009, 872-879.

18 D. A. Fletcher, R. F. McMeeking and D. Parkin, J. Chem. Inf. Comput. Sci., 1996, 36, 746-749.

19 A. S. Potapov and A. I. Khlebnikov, Polyhedron, 2006, 25, 2683-2690.
20 P. Kübler and J. Sundermeyer, Dalton Trans., 2014, 43, 3750-3766.

21 G. M. Sheldrick, Acta Cryst., 2015, A71, 3.

22 O. V. Dolomanov, L. J. Bourhis, R. J. Gildea, J. A. K. Howard and H. Puschmann, J. Appl. Crystallogr., 2009, 42, 339341. 